\title{
PRIMITIVE CENTRAL IDEMPOTENTS OF FINITE GROUP RINGS OF SYMMETRIC GROUPS
}

\author{
HARALD MEYER
}

\begin{abstract}
Let $p$ be a prime. We denote by $S_{n}$ the symmetric group of degree $n$, by $A_{n}$ the alternating group of degree $n$ and by $\mathbb{F}_{p}$ the field with $p$ elements. An important concept of modular representation theory of a finite group $G$ is the notion of a block. The blocks are in one-to-one correspondence with block idempotents, which are the primitive central idempotents of the group ring $\mathbb{F}_{q} G$, where $q$ is a prime power. Here, we describe a new method to compute the primitive central idempotents of $\mathbb{F}_{q} G$ for arbitrary prime powers $q$ and arbitrary finite groups $G$. For the group rings $\mathbb{F}_{p} S_{n}$ of the symmetric group, we show how to derive the primitive central idempotents of $\mathbb{F}_{p} S_{n-p}$ from the idempotents of $\mathbb{F}_{p} S_{n}$. Improving the theorem of Osima for symmetric groups we exhibit a new subalgebra of $\mathbb{F}_{p} S_{n}$ which contains the primitive central idempotents. The described results are most efficient for $p=2$. In an appendix we display all primitive central idempotents of $\mathbb{F}_{2} S_{n}$ and $\mathbb{F}_{4} A_{n}$ for $n \leq 50$ which we computed by this method.
\end{abstract}

\section{INTRODUCTION AND NOTATION}

Let $p$ be a prime, let $q=p^{s}$ for some $s \in \mathbb{N}$ and let $G$ be a finite group. For the finite field with $q$ elements we write $\mathbb{F}_{q}$, and $\mathbb{F}_{q} G$ denotes the group ring of $G$ over $\mathbb{F}_{q}$. We use $S_{n}$ and $A_{n}$ for the symmetric and alternating group of degree $n$, respectively. We write $\mathbb{Z}_{m}$ for a cyclic group of order $m$, and $\mathbb{Z}_{m} 2 S_{i}$ for the wreath product of this group with $S_{i}$. We use $\mathrm{C}_{G}(g)$ for the centralizer of $g \in G$, and $\mathrm{C}_{G}(U)$ for the centralizer of $U \subseteq G$. The centre of a group ring $F G$ is denoted by $Z(F G)$ and the radical of the centre by $\operatorname{Rad}(Z(F G))$. We use $\operatorname{Irr} G$ for the set of irreducible characters of $G$ defined over the field $\mathbb{C}$ of complex numbers and we write $\operatorname{ord}(g)$ for the order of an element $g \in G$. The symbols $\mathbb{Z}$ and $\mathbb{N}$ denote the integers and the natural numbers, respectively.

A big part of modular representation theory deals with blocks. There are several possibilities to characterize blocks, for instance by the block idempotents, i.e. the primitive central idempotents of $\mathbb{F}_{q} G$. Therefore it is important to find methods to compute the primitive central idempotents.

The usual method to compute primitive central idempotents is described in [8], Lemma 16.6. For this method it is necessary to compute the character table of $G$ over the field $\mathbb{C}$ of complex numbers first. For the symmetric group $S_{50}$ it is known that there are 204226 characters over $\mathbb{C}$, but there are only 5 primitive central idempotents of $\mathbb{F}_{2} S_{50}$. Due to the vast amount of data it is not possible to compute

Received by the editor December 15, 2006 and, in revised form, March 8, 2007.

2000 Mathematics Subject Classification. Primary 20C05, 20C30, 20C40.

Key words and phrases. Group ring, symmetric group, primitive central idempotent.

(c)2007 American Mathematical Society Reverts to public domain 28 years from publication 
the character table of $S_{50}$, but it is possible to compute the 5 primitive central idempotents of $\mathbb{F}_{2} S_{50}$ using the algorithm described in [12, 2.21. Thus we use the first part to give a description of this algorithm, which works for all finite group rings.

The specialization to symmetric and alternating groups allows us to speed up the algorithm. We do this by proving theoretical results about the group rings $\mathbb{F}_{p} S_{n}$ and their primitive central idempotents. To state the results we need the following notation: It is well known that the conjugacy classes of $S_{n}$ can be indexed by the partitions of $n$. We write $\mu=1^{\alpha_{1}}, \ldots, n^{\alpha_{n}}$ for the partition

$$
\mu=(\underbrace{1, \ldots, 1}_{\alpha_{1}}, \underbrace{2, \ldots, 2}_{\alpha_{2}}, \ldots)
$$

of $n$. The fact that $\mu$ is a partition of $n$ is abbreviated by $\mu \vdash n . C_{\mu}$ is the conjugacy class of $S_{n}$ belonging to $\mu$, and $C_{\mu}^{+}$denotes the class sum of $C_{\mu}$ in $\mathbb{F}_{p} S_{n}$. The $n$-tuple of multiplicities $\left(\alpha_{1}, \ldots, \alpha_{n}\right)$ appearing in $\mu$ is called the cycle type of the element $\sigma \in C_{\mu}$. We define

$$
W(\mu):=\sum_{i=2}^{n} i \cdot \alpha_{i}
$$

and call it the essential weight of the partition $\mu$. For our purpose it is convenient to ignore the parts equal to 1 in the partition, because an element like $(1,2,3) \in S_{3}$ is also an element of bigger symmetric groups. So we usually write $\mu=2^{\alpha_{2}}, \ldots, n^{\alpha_{n}}$ for a partition and the corresponding class $C_{\mu}$ is a class of an arbitrary symmetric group $S_{n}$ with $n \geq W(\mu)$ depending on the context, i.e. $C_{2}$ denotes the conjugacy class of transpositions in every symmetric group $S_{n}, n \geq 2$. If we want to emphasize that $C_{\mu}$ is a class of a certain symmetric group $S_{n}$ we write $\left.C_{\mu}\right|_{S_{n}}$ and also $\left.C_{\mu}^{+}\right|_{S_{n}}$ for the class sum in $\mathbb{F}_{p} S_{n}$. The class multiplication coefficients $c_{\lambda \mu \nu} \in \mathbb{F}_{p}$ are defined via

$$
C_{\lambda}^{+} C_{\mu}^{+}=\sum_{\nu \vdash n} c_{\lambda \mu \nu} C_{\nu}^{+} .
$$

Here, the $c_{\lambda \mu \nu}$ depend on $n$, but to keep notation simple we usually suppress the $n$. In cases of ambiguity we write $\left.c_{\lambda \mu \nu}\right|_{S_{n}}$ for the coefficient of $\left.C_{\nu}^{+}\right|_{S_{n}}$ in $\left.\left.C_{\lambda}^{+}\right|_{S_{n}} \cdot C_{\mu}^{+}\right|_{S_{n}}$.

For an element

$$
B=\sum_{g \in G} a_{g} g
$$

of a group ring $\mathbb{F}_{q} G$ the support is the set $\operatorname{supp} B:=\left\{g \in G \mid a_{g} \neq 0\right\}$. The theorem of Osima mentioned above states that the support of primitive central idempotents of $\mathbb{F}_{q} G$ consists of $p^{\prime}$-elements, i.e. elements of an order which is not divisible by $p$. Usually these elements and the corresponding class sums are called $p$-regular, but in symmetric groups this expression is used for partitions $\mu=1^{\alpha_{1}}, \ldots, n^{\alpha_{n}}$, where $\alpha_{i}<p$ for $i=1, \ldots, n$. Thus we avoid using this expression for the classes and use the term ' $p$ '-conjugacy class' instead for the conjugacy classes of $p^{\prime}$-elements. The corresponding partitions are usually called $p$-class regular, but we prefer to call them $p^{\prime}$-partitions. Furthermore we define $p$-near-regular partitions and conjugacy classes: $\mu=1^{\alpha_{1}}, \ldots, n^{\alpha_{n}}$ is called $p$-near-regular, when $\alpha_{i}<p$ for $i=2, \ldots, n$ and the corresponding conjugacy classes are the $p$-near-regular classes. A theorem of Murray (14, Corollary 5) states that the vector space

$$
\left.Z_{p^{\prime}}^{G}:=\left\langle C^{+}\right| C \text { is } p^{\prime}-\text { conjugacy class of } G\right\rangle
$$


is a subalgebra of the centre of $\mathbb{F}_{q} G$, if $G$ is a symmetric group. We also use the vector spaces

$$
\left.Z_{p-r e g}^{S_{n}}:=\left\langle C_{\mu}^{+}\right| \mu \text { is } p-\text { regular partition of } n\right\rangle
$$

and

$$
\left.Z_{p-n r e g}^{S_{n}}:=\left\langle C^{+}\right| C \text { is } p-\text { near-regular conjugacy class of } S_{n}\right\rangle .
$$

Now we can state our main theorems. Section 2 is devoted to proving the following result.

Theorem 1. Let $m<n$ and $m \equiv n \bmod p$. Let $\delta$ be the homomorphism of vector spaces defined by

$$
\delta: Z_{p^{\prime}}^{S_{n}} \longrightarrow Z_{p^{\prime}}^{S_{m}}, \delta\left(C_{\mu}^{+}\right):=\left\{\begin{array}{cl}
\left.C_{\mu}^{+}\right|_{S_{m}}, & \text { if } W(\mu) \leq m \\
0, & \text { if } W(\mu)>m .
\end{array}\right.
$$

Then $\delta$ is a homomorphism of algebras.

Let $e_{1}, \ldots, e_{r}$ denote the primitive central idempotents of $\mathbb{F}_{p} S_{n}$. Then $\delta$ has the following properties:

1) If $\delta\left(e_{i}\right) \neq 0$, then $\delta\left(e_{i}\right)$ is a primitive central idempotent of $\mathbb{F}_{p} S_{m}$.

2) For every primitive central idempotent $f$ of $\mathbb{F}_{p} S_{m}$ there is an $i \in\{1, \ldots, r\}$ such that $f=\delta\left(e_{i}\right)$.

We remark that our Theorem 1 is related to Theorem 1.6 of [16].

In Section 3 we prove the following two theorems:

Theorem 2. $Z_{p^{\prime}}^{S_{n}} \cap Z_{p-n r e g}^{S_{n}}$ is an algebra.

Theorem 3. The primitive central idempotents of $\mathbb{F}_{p} S_{n}$ are contained in $Z_{p^{\prime}}^{S_{n}} \cap$ $Z_{p-n r e g}^{S_{n}}$, i.e. the support $\operatorname{supp} e_{i}$ of a primitive central idempotent $e_{i}$ of $\mathbb{F}_{p} S_{n}$ consists of p-near-regular $p^{\prime}$-conjugacy classes.

These theorems help to speed up the program, so we were able to compute the primitive central idempotents of $\mathbb{F}_{2} S_{n}$ and $\mathbb{F}_{4} A_{n}$ for $n \leq 50$ using the computer algebra package GAP [2] and a program written in SYMMETRICA [10] provided by A. Kohnert. The Appendix contains the computational results.

We note that there is a theoretical result which provides one of the primitive central idempotents of the group rings $\mathbb{F}_{2} S_{n}$, if $n$ is of the form $n=\frac{m(m+1)}{2}$ with an integer $m$. R. Gow proves in Theorem 3 of 3 ] that in this case one of the primitive central idempotents of $\mathbb{F}_{2} S_{n}$ has the form $e=C^{+}$, where $C$ is the conjugacy class of elements corresponding to the partition $(2 m-1,2 m-5,2 m-9, \ldots)$ of $n$. We don't use this result for our computations for symmetric groups, but we use it to determine the primitive central idempotents of $\mathbb{F}_{4} A_{n}$, because Gow also proves that this idempotent is the only idempotent of $\mathbb{F}_{2} S_{n}$ which splits in $\mathbb{F}_{4} A_{n}$.

\section{Computation of primitive Central idempotents OF FINITE GROUP RINGS}

Let $F$ be a splitting field for $G$ of characteristic $p>0$. We already mentioned that the usual method for computing the primitive central idempotents of a group ring $F G$ uses the character table of $G$ over $\mathbb{C}$. But what if we do not know the character table? Can we compute the idempotents within $F G$ ? The first observation is that we can assume $F$ to be finite because every group has a finite splitting field of characteristic $p$ according to a theorem of Brauer ([6], Theorem VII.2.6). Now if 
$F G$ is finite and $B \in Z(F G)$, then the sequence $\left(B^{n}\right)_{n \in \mathbb{N}}$ has to be periodic, i.e. there exist $r, m \in \mathbb{N}$ such that $B^{r}=B^{r+m}$. This idea can be used to construct central idempotents and leads to Algorithm 7 w which is already described in [12. The following theorem ([12, Satz 2.1) is the foundation of the algorithm. As we will need it in section 2 , we prove it here again. Now let $F$ be an arbitrary finite field of characteristic $p>0$ and let $\bar{F}$ denote the algebraic closure of $F$. We will mention it explicitly if we assume $F$ to be a splitting field for $G$ or for $Z(F G)$.

Theorem 4. Let $B \in Z(F G)$ and let $m \in \mathbb{N}$ be such that $B^{r}=B^{r+m}$, for all $r \in \mathbb{N}$ suitable large. Choose $r=l \cdot m$, where $l \neq 0$ and suppose that $m=p^{s} \cdot d$, where $s \geq 0$ and $p \nmid d$. Let $\zeta \in \bar{F}$ be a primitive $d$-th root of unity and put

$$
f_{k}:=d^{-1} \sum_{i=0}^{d-1}\left(\zeta^{k}\right)^{i} B^{r+p^{s} \cdot i}
$$

for $0 \leq k \leq d-1$. Then $f_{k}=0$ or $f_{k}$ is a central idempotent of $F\left(\zeta^{k}\right) G$, the group ring of $G$ over the field $F\left(\zeta^{k}\right)$. For $k \neq n$ we have $f_{k} f_{n}=0$ in $F(\zeta) G$. If $B^{r} \neq 0$, then there is a $k$ such that $f_{k} \neq 0$ and $B^{r}$ is a central idempotent itself.

Proof. For $0 \leq k \leq d-1$ we define

$$
D_{k}:=\sum_{i=0}^{d-1}\left(\zeta^{k}\right)^{i} \cdot B^{r+p^{s} \cdot i}
$$

Now let $w:=\zeta^{k}$. Then we have $w^{d}=1$ and get

$$
w^{j} B^{r+p^{s} \cdot j} \cdot D_{k}=\sum_{i=0}^{d-1} w^{i+j} B^{r+l m+p^{s} \cdot(i+j)}=D_{k}
$$

using the periodicity of the sequence $\left(B^{n}\right)_{n \in \mathbb{N}}$. Hence we obtain $D_{k}^{2}=d \cdot D_{k}$, so $f_{k}=d^{-1} D_{k}$ is 0 or a central idempotent in $F\left(\zeta^{k}\right) G$.

If $0 \leq n \leq d-1$ and $n \neq k$, then

$$
D_{k} D_{n}=\sum_{i=0}^{d-1}\left(\zeta^{k}\right)^{i}\left(\zeta^{n}\right)^{-i}\left[\left(\zeta^{n}\right)^{i} B^{r+p^{s} \cdot i} D_{n}\right]=D_{n} \cdot \sum_{i=0}^{d-1}\left(\zeta^{k-n}\right)^{i}=0 .
$$

Therefore the $f_{k}$ with $f_{k} \neq 0$ are orthogonal idempotents.

Finally, if $B^{r} \neq 0$, then

$$
\sum_{k=0}^{d-1} D_{k}=d B^{r} \neq 0
$$

i.e. there is an $f_{k} \neq 0$. As the $f_{k}$ are orthogonal we obtain

$$
\left(B^{r}\right)^{2}=\left(\sum_{k=0}^{d-1} f_{k}\right)^{2}=\sum_{k=0}^{d-1} f_{k}^{2}=\sum_{k=0}^{d-1} f_{k}=B^{r} .
$$

A similar computation leads to the following corollary ([12, 2.3):

Corollary 5. Let $e_{1}, \ldots, e_{r}$ be the primitive central idempotents of $F G$. Then the span

$$
\left\langle e_{1}, \ldots, e_{r}\right\rangle_{F}=\left\{B \in Z(F G) \mid B^{|F|}=B\right\} .
$$

The following theorem (see [12], Satz 2.17) is the foundation for our algorithm: 
Theorem 6. Let $F$ be a splitting field for $Z(F G)$. Let $C_{1}, \ldots, C_{k}$ be the $p^{\prime}$-conjugacy classes of $G$ and let $e_{1}, \ldots, e_{r}$ be the primitive central idempotents of $F G$. Then there is an $n_{0} \in \mathbb{N}$ such that

$$
\left\langle e_{1}, \ldots, e_{r}\right\rangle_{F}=\left\langle\left(C_{1}^{+}\right)^{p^{n}}, \ldots,\left(C_{k}^{+}\right)^{p^{n}}\right\rangle_{F}
$$

for all $n \geq n_{0}$.

Proof. Let $C_{k+1}, \ldots, C_{c}$ be the $p$-singular conjugacy classes of $G$, i.e. the classes of elements whose order is divisible by $p$. As the class sums $C_{1}^{+}, \ldots, C_{c}^{+}$form a basis of $Z(F G)$, we get

$$
\left\langle e_{1}, \ldots, e_{r}\right\rangle_{F}=\left\langle\left(C_{1}^{+}\right)^{p^{n}}, \ldots,\left(C_{c}^{+}\right)^{p^{n}}\right\rangle_{F}
$$

for all $n \in \mathbb{N}$ suitable large by [11, p. 434 (an elementary proof can be found in [12, Satz 2.11). According to the theorem of Osima (8, Theorem 23.6) we know $\left\langle e_{1}, \ldots, e_{r}\right\rangle_{F} \subset\left\langle C_{1}^{+}, \ldots, C_{k}^{+}\right\rangle_{F}$. Now let $n_{0}$ be a multiple of $|F|$, which is suitably large. Then

$$
\varphi: Z(F G) \longrightarrow Z(F G), B \longmapsto B^{p^{n_{0}}}
$$

is a homomorphism of vector spaces, hence we obtain

$$
\begin{aligned}
\left\langle e_{1}, \ldots, e_{r}\right\rangle_{F} & =\varphi\left(\left\langle e_{1}, \ldots, e_{r}\right\rangle_{F}\right) \subset\left\langle\left(C_{1}^{+}\right)^{p^{n_{0}}}, \ldots,\left(C_{k}^{+}\right)^{p^{n_{0}}}\right\rangle_{F} \\
& \subset\left\langle\left(C_{1}^{+}\right)^{p^{n_{0}}}, \ldots,\left(C_{c}^{+}\right)^{p^{n_{0}}}\right\rangle_{F}=\left\langle e_{1}, \ldots, e_{r}\right\rangle .
\end{aligned}
$$

Thus we get our statement for all $n \geq n_{0}$.

This leads to the following algorithm for computing primitive central idempotents of finite group rings, which can be found in [12, 2.21. But the algorithm in 12 contains some minor gaps, which we fill here.

Algorithm 7. Let $G$ be a finite group and $p$ a prime. Let $F$ be a splitting field for $Z(F G)$ with char $F=p$. The computation of the primitve central idempotents of $F G$ can be accomplished by the following steps:

1) Compute the $p^{\prime}$-conjugacy classes $C_{1}, \ldots, C_{b}$ of $G$.

2) For $i=1, \ldots, b$, do the following: By computing successive powers of the class sum $C_{i}^{+}$, determine the minimal integers $r_{i}, m_{i} \geq 1$ such that $\left(C_{i}^{+}\right)^{r_{i}}=$ $\left(C_{i}^{+}\right)^{r_{i}+m_{i}}$, and $r_{i}$ is a multiple of $m_{i}$. Write $m_{i}=p^{s_{i}} \cdot d_{i}$, where $s_{i} \geq 0$ and $p \nmid d_{i}$.

3) Compute the idempotents

$$
f_{k}^{(i)}:=d_{i}^{-1} \sum_{j=0}^{d_{i}-1}\left(\zeta_{i}^{k}\right)^{j}\left(C_{i}^{+}\right)^{r_{i}+p^{s_{i} \cdot j}}
$$

for $1 \leq i<b$ and $0 \leq k \leq d_{i}-1$, where $\zeta_{i}$ denotes a primitive $d_{i}$-th root of unity in a suitable extension of the field $\mathbb{F}_{p}$.

4) Choose a basis $f_{1}, \ldots, f_{r}$ of the mostly linear dependent set

$$
\left\{f_{k}^{(i)} \mid 1 \leq i \leq b, 0 \leq k \leq d_{i}-1\right\} \text {. }
$$

If $r=1$, then $f_{1}=1$ is the only central idempotent of $F G$ and the computation is finished. For $r>1$ we have to accomplish one more step:

5) For $i=1, \ldots, r-1 d o$ :

$$
\{\text { For } j=i+1, \ldots, r \text { do: }
$$




$$
\begin{aligned}
& \left\{\text { Set } w:=f_{i}\right. \text {. } \\
& \text { If } f_{i} \cdot f_{j}=f_{j} \text { then } \\
& \text { else } \\
& \left\{\text { set } f_{i}:=f_{j}, f_{j}:=w, w:=f_{i} .\right\} \\
& \left\{\text { If } f_{i} \cdot f_{j} \neq 0 \text { then }\left\{\text { replace } f_{i} \text { with } f_{i} \cdot f_{j} .\right\}\right. \\
& \text { If } \operatorname{dim}\left\langle f_{1}, \ldots, f_{r}\right\rangle<r \text { then } \\
& \{k:=j \text {. } \\
& \text { While } \operatorname{dim}\left\langle f_{1}, \ldots, f_{k-1}, w, f_{k+1}, \ldots, f_{r}\right\rangle<r d o \\
& \{k:=k+1 .\} \\
& f_{k}:=w . \\
& \}
\end{aligned}
$$

The elements $f_{1}, \ldots, f_{r}$ are the primitive central idempotents of $F G$.

Remark. 1) It is not necessary to compute a splitting field for $Z(F G)$ in advance, as the $\zeta_{i}$ arise during the computation. In general, $\mathbb{F}_{p}\left(\zeta_{1}, \ldots, \zeta_{k}\right)$ is not a splitting field for $G$, but it is a splitting field for the centre $Z(F G)$ of the group ring $F G$ : Indeed it is the minimal splitting field of characteristic $p$ for $Z(F G)$. A proof can be found in 12, Satz 3.16.

2) Further knowledge about the group ring $F G$ can be used to speed up the program. For example an automorphism $\alpha$ of $Z(F G)$ can be applied in step 2 of the algorithm to deduce the powers of $\alpha\left(C^{+}\right)$very fast, if the powers of $C^{+}$are already computed.

This remark together with a previous lemma and the decomposition

$$
Z(F G)=\left\langle f_{1}, \ldots, f_{r}\right\rangle_{F} \oplus \operatorname{Rad}(Z(F G))
$$

of $Z(F G)$ as a vector space ([8], Lemma 25.1) allows us to compute generators of $\operatorname{Rad}(Z(F G))$ as a vector space:

Theorem 8. Let $F$ be a finite splitting field of characteristic $p$ for $Z(F G)$ with $|F|=p^{n}$. Let $C_{1}, \ldots, C_{c}$ be the conjugacy classes of $G$ (here we need them all). Then

$$
\operatorname{Rad}(Z(F G))=\left\langle C_{1}^{+}-\left(C_{1}^{+}\right)^{p^{n}}, \ldots, C_{c}^{+}-\left(C_{c}^{+}\right)^{p^{n}}\right\rangle \text { (as a vector space). }
$$

Proof. Let $e_{1}, \ldots, e_{r}$ be the primitive central idempotents of $F G$. Let $i \in\{1, \ldots, c\}$. According to [1], p. 434, there is an $m \in \mathbb{N}$ with $\left(C_{i}^{+}\right)^{p^{m}} \in\left\langle e_{1}, \ldots, e_{r}\right\rangle$. Using Corollary 5 we obtain

$$
\left(C_{i}^{+}-\left(C_{i}^{+}\right)^{p^{n}}\right)^{p^{m}}=\left(C_{i}^{+}\right)^{p^{m}}-\left(\left(C_{i}^{+}\right)^{p^{m}}\right)^{p^{n}}=\left(C_{i}^{+}\right)^{p^{m}}-\left(C_{i}^{+}\right)^{p^{m}}=0
$$

and thus $C_{i}^{+}-\left(C_{i}^{+}\right)^{p^{n}} \in \operatorname{Rad}(Z(F G))$. 
Now we consider the map

$$
\varphi: Z(F G) \longrightarrow Z(F G), \varphi(x):=x-x^{p^{n}} .
$$

$\varphi$ is a homomorphism of vector spaces. According to Corollary 5 the kernel is $\left\langle e_{1}, \ldots, e_{r}\right\rangle$. The image is a subset of $\operatorname{Rad}(Z(F G))$. By [8], Lemma 25.1, we obtain our statement.

Remark 9. We keep the notation of the last theorem. The proof of the preceding theorem also provides a method to compute the projection of an arbitrary element $B \in Z(F G)$ to the vector spaces $\left\langle e_{1}, \ldots, e_{r}\right\rangle_{F}$ and $\operatorname{Rad}(Z(F G))$ : Choose $m=k \cdot n$, such that $B^{p^{m}} \in\left\langle e_{1}, \ldots, e_{r}\right\rangle$ (this is true if $\left.p^{m}>c^{p^{n}}\right)$. Then $B-B^{p^{m}} \in \operatorname{Rad}(Z(F G))$ and $B^{p^{m}}$ and $B-B^{p^{m}}$ are the projections of $B$ to $\left\langle e_{1}, \ldots, e_{r}\right\rangle_{F}$ and $\operatorname{Rad}(Z(F G))$, respectively.

In the following sections we will see how we can improve the algorithm for symmetric groups.

\section{Connections BetweEn PRIMitive CENTRAL IDEMPotents OF $\mathbb{F}_{p} S_{n}$ FOR DIFFERENT $n$}

The methods developed in the first section work for all finite groups and fields of characteristic $p$, even in the case where $p \nmid|G|$. But to apply the algorithm successfully to bigger symmetric groups we have to speed up the algorithm. In the algorithm we have to multiply sums of class sums. How can we do that quickly? If $C_{1}, \ldots, C_{m}$ are the conjugacy classes of $S_{n}$, then we get

$$
C_{i}^{+} C_{j}^{+}=\sum_{k=1}^{m} c_{i j k} C_{k}^{+}
$$

with coefficients $c_{i j k} \in \mathbb{F}_{p}$. According to [4], Theorem 4.6, we know that

$$
c_{i j k} \equiv \frac{\left|C_{i} \| C_{j}\right|}{\left|S_{n}\right|} \sum_{\chi \in \operatorname{Irr} S_{n}} \chi\left(g_{i}\right) \chi\left(g_{j}\right) \frac{\chi\left(g_{k}^{-1}\right)}{\chi(1)} \bmod p,
$$

where $g_{i} \in C_{i}$ for $i \in\{1, \ldots, m\}$. So we can compute the class multiplication coefficients $c_{i j k}$, if we know some parts of the character table of $S_{n}$. Character values for symmetric groups can be computed very fast with the Murnaghan-Nakayama formula, see [7, 2.4.7, even for $S_{50}$. But the number of class multiplication coefficients we have to compute must not be too big, if we want to get results for a group like $S_{50}$. So we need theoretical results to reduce the work. A first result in this direction is a theorem of Murray ([14], Corollary 5): $Z_{p^{\prime}}^{S_{n}}$ is an algebra. So if we multiply class sums of $p^{\prime}$-conjugacy classes we only need to compute the coefficients of $p^{\prime}$-class sums. Due to Algorithm 7 we only need to consider $p^{\prime}$-conjugacy classes, so we can use this result for all our computations. Here and in the following sections we will prove some more theorems which allow us to reduce the number of coefficients we need to compute.

The computations are simplified by the fact that $\mathbb{F}_{p}$ is a splitting field for $S_{n}$ according to [7, 2.1.12, so we can choose $F=\mathbb{F}_{p}$. 
Lemma 10. Let $n>p$ and let $\delta_{n}$ be the homomorphism of vector spaces defined by

$$
\delta_{n}: Z_{p^{\prime}}^{S_{n}} \longrightarrow Z_{p^{\prime}}^{S_{n-p}}, C_{\mu}^{+} \longmapsto\left\{\begin{array}{cl}
\left.C_{\mu}^{+}\right|_{S_{n-p}}, & \text { if } W(\mu) \leq n-p, \\
0, & \text { if } W(\mu)>n-p .
\end{array}\right.
$$

Then $\delta_{n}$ is a homomorphism of algebras.

Proof. We write $S_{n-p}$ for the subgroup of $S_{n}$ containing the permutations, which fix the numbers $n-p+1, \ldots, n$. Let $\tau:=(n-p+1, \ldots, n) \in S_{n}$. Then for the centralizer we get $\mathrm{C}_{S_{n}}(\tau)=S_{n-p} \times\langle\tau\rangle$. Now let

$$
\operatorname{Br}_{\langle\tau\rangle}: Z\left(\mathbb{F}_{p} S_{n}\right) \longrightarrow Z\left(\mathbb{F}_{p} \mathrm{C}_{S_{n}}(\tau)\right)
$$

be the Brauer-homomorphism (see [15, Theorem 4.9). $\mathrm{Br}_{\langle\tau\rangle}$ is a homomorphism of algebras with $\operatorname{Br}_{\langle\tau\rangle}\left(C_{\mu}^{+}\right)=\left(C_{\mu} \cap \mathrm{C}_{S_{n}}(\tau)\right)^{+}$. Thus $\delta_{n}$ is the restriction of $\operatorname{Br}_{\langle\tau\rangle}$ to $Z_{p^{\prime}}^{S_{n}}$, and is therefore a homomorphism of algebras, by [14, Corollary 5].

Remark 11. If we define the homomorphism $\delta_{n}$ of the preceding lemma for $Z\left(\mathbb{F}_{p} S_{n}\right)$ we usually do not get a homomorphism of algebras: If $C_{\tau}$ denotes the conjugacy class of $\tau$ in $S_{n}$, then $C_{\tau} \cap \mathrm{C}_{S_{n}}(\tau)$ is not contained in the subgroup $S_{n-p}$ of $\mathrm{C}_{S_{n}}(\tau)$ and the same problem occurs for conjugacy classes of partitions containing $p$. For example for the conjugacy class $C_{2}$ of transpositions it is easy to see that $\left(C_{2}^{+}\right)^{2}=$ $C_{1}^{+}+C_{3}^{+}$for $n \equiv 2,3 \bmod 4$ and $\left(C_{2}^{+}\right)^{2}=C_{3}^{+}$for $n \equiv 0,1 \bmod 4$ in $\mathbb{F}_{2} S_{n}, n \geq 3$.

Now we can prove our Theorem 1 .

Proof of Theorem 1. We keep the notation of Lemma 10. If $m=n-k \cdot p$, then $\delta$ is the composition of $\delta_{n}, \delta_{n-p}, \ldots, \delta_{n-(k-1) p}$ and thus is a homomorphism of algebras. Therefore $\left\{\delta\left(e_{1}\right), \ldots, \delta\left(e_{r}\right)\right\} \backslash\{0\}$ is a set of central orthogonal idempotents. We prove the remaining statements in several steps:

1) For every central idempotent $f \in Z\left(\mathbb{F}_{p} S_{m}\right)$ there is a central idempotent $e \in Z\left(\mathbb{F}_{p} S_{n}\right)$ with $\delta(e)=f$ :

We define a homomorphism $\Delta$ of vector spaces by

$$
\Delta: Z\left(\mathbb{F}_{p} S_{m}\right) \longrightarrow Z\left(\mathbb{F}_{p} S_{n}\right),\left.\left.C_{\mu}^{+}\right|_{S_{m}} \mapsto C_{\mu}^{+}\right|_{S_{n}}
$$

and put $F:=\Delta(f)$. Now let $r, m \in \mathbb{N}$ such that $F^{r}=F^{r+m}$ and $r=l \cdot m$ for an $l \in \mathbb{N}$. Let $m=p^{s} \cdot d$ with $p \nmid d$. We set

$$
e:=d^{-1} \sum_{i=0}^{d-1} F^{r+p^{s} \cdot i}
$$

Then $e$ is 0 or a central idempotent of $\mathbb{F}_{p} S_{n}$ by Theorem 4. We obtain

$$
\delta(e)=d^{-1} \sum_{i=0}^{d-1} \delta(F)^{r+p^{s} \cdot i}=d^{-1} \sum_{i=0}^{d-1} f=f \neq 0,
$$

so $e \neq 0$ and $\delta(e)=f$.

2) For every primitive central idempotent $f \in Z\left(\mathbb{F}_{p} S_{m}\right)$ there is $i \in\{1, \ldots, r\}$ with $f=\delta\left(e_{i}\right)$ :

By 1$)$ there exists a central idempotent $e$ of $Z\left(\mathbb{F}_{p} S_{n}\right)$ with $\delta(e)=f$. Now let $e=\sum_{i=1}^{k} e_{i}$ be the decomposition of $e$ in primitive central idempotents of 
$Z\left(\mathbb{F}_{p} S_{n}\right)$. Then we have

$$
f=\delta(e)=\sum_{i=1}^{k} \delta\left(e_{i}\right),
$$

and as $\delta$ is a homomorphism of algebras, the $\delta\left(e_{i}\right)$ are 0 or central orthogonal idempotents of $Z\left(\mathbb{F}_{p} S_{m}\right)$. But $f$ is primitive, therefore there is an $i_{0} \in$ $\{1, \ldots, k\}$ such that $\delta\left(e_{i_{0}}\right) \neq 0, \delta\left(e_{j}\right)=0$ for $j \neq i_{0}$ and we obtain $f=\delta\left(e_{i_{0}}\right)$.

3) If $e \in\left\{e_{1}, \ldots, e_{r}\right\}$ and $\delta(e)=f \neq 0$, then $f$ is a primitive central idempotent of $\mathbb{F}_{p} S_{m}$ :

We assume that $f$ is not primitive as a central idempotent. Then let $f=\sum_{i=1}^{k} f_{i}$ be a decomposition of $f$ in primitive central idempotents. By 2) there are primitive central idempotents $e_{1}, \ldots, e_{k}$ of $\mathbb{F}_{p} S_{n}$ with $\delta\left(e_{i}\right)=f_{i}$. As $\delta(e)=f \neq f_{1}=\delta\left(e_{1}\right)$ we have $e \neq e_{1}$. Now both $e$ and $e_{1}$ are different primitive central idempotents of $\mathbb{F}_{p} S_{n}$, so they are orthogonal. Hence we obtain

$$
0=\delta\left(e \cdot e_{1}\right)=\delta(e) \cdot \delta\left(e_{1}\right)=f \cdot f_{1}=f_{1},
$$

a contradiction.

Theorem 1 allows us to write down the primitive central idempotents of $\mathbb{F}_{p} S_{n}$ in a very compact way: For example, it will do to write down the primitive central idempotents of $\mathbb{F}_{2} S_{50}$ and $\mathbb{F}_{2} S_{49}$ to know the primitive central idempotents of $\mathbb{F}_{2} S_{n}$ for all $n \leq 50$. Furthermore our computations in connection with Theorem 1 lead to statements like the following:

$C_{11} \notin \operatorname{supp} e$ for all primitive central idempotents $e$ of $\mathbb{F}_{2} S_{n}, n \in \mathbb{N}$,

because $C_{11}$ is neither included in the support of the primitive central idempotents of $\mathbb{F}_{2} S_{11}$ nor in the according support in $\mathbb{F}_{2} S_{12}$.

For the computation of idempotents the most important part of Theorem 1 is the fact that $\delta$ is a homomorphism of algebras because this provides the following corollary:

Corollary 12. Let $\lambda, \mu, \nu$ be $p^{\prime}$-partitions of $n$ with $W(\mu) \geq W(\lambda)$. Let $m \equiv n$ $\bmod p$ be minimal with $W(\nu) \leq m$. Then for the class multiplication coefficient $\left.c_{\lambda \mu \nu}\right|_{S_{n}}$ we have

$$
\left.c_{\lambda \mu \nu}\right|_{S_{n}}=\left\{\begin{array}{cl}
0, & \text { if } W(\nu) \leq W(\mu)-p \text { or } W(\nu)>W(\mu)+W(\lambda), \\
\left.c_{\lambda \mu \nu}\right|_{S_{m}}, & \text { if } W(\mu)-p<W(\nu) \leq W(\mu)+W(\lambda) .
\end{array}\right.
$$

Proof. If $\pi \in C_{\lambda}$ and $\sigma \in C_{\mu}$, then $\pi$ moves $W(\lambda)$ points and $\sigma$ moves $W(\mu)$ points, so $\pi \sigma$ moves at most $W(\lambda)+W(\mu)$ points. Thus if $W(\nu)>W(\lambda)+W(\mu)$, then obviously $\left.c_{\lambda \mu \nu}\right|_{S_{n}}=0$. Now let $W(\nu) \leq W(\mu)-p$ and let $\delta_{n}$ be as in Lemma 10. As $\delta_{n}$ is a homomorphism and $\delta_{n}\left(C_{\mu}^{+}\right)=0$, but $\delta_{n}\left(C_{\nu}^{+}\right) \neq 0$, it follows that $\left.c_{\lambda \mu \nu}\right|_{S_{n}}=0$, in this case. The remaining statement is clear by applying $\delta_{n}$.

\section{A further SUbalgebra of $\mathbb{F}_{p} S_{n}$ CONTAining the idempotents}

To prove Theorem 2 we need the following version of a lemma proved in [1], which is just a formulation of the statement that the Brauer homomorphism is an algebra homomorphism. Additionally it is a formulation of another theorem of Osima ([15, Theorem 4.1]): If $C$ is a conjugacy class of $G$ and $g \in C$, then a 
Sylow-p-subgroup of $\mathrm{C}_{G}(g)$ is called a defect group of $C$. If $Z_{D}(F G)$ denotes the $F$-span of all class sums $C^{+}$such that the defect groups of $C$ are contained in a $G$-conjugate of a certain $p$-subgroup $D$ of $G$, then $Z_{D}(F G)$ is an ideal of $Z(F G)$. But Lemma 13 is more detailed specifying elements and making a statement about the Sylow- $p$-subgroups of these elements. In the proof of Theorem 2 we apply this more detailed version of the theorem of Osima.

Lemma 13. Let $G$ be a finite group. Let $C_{1}, \ldots, C_{s}$ be the conjugacy classes of $G$ and let $C_{1}^{+}, \ldots, C_{s}^{+}$be the corresponding class sums in $\mathbb{Z} G$. Now let

$$
C_{i}^{+} C_{j}^{+}=\sum_{k=1}^{s} a_{i j k} C_{k}^{+}
$$

If $a_{i j k} \not \equiv 0 \bmod p$, then for every element $z \in C_{k}$ and every Sylow-p-subgroup $P_{z}$ of $\mathrm{C}_{G}(z)$ there are elements $x \in C_{i}$ and $y \in C_{j}$ as well as Sylow-p-subgroups $P_{x}$ of $\mathrm{C}_{G}(x)$ and $P_{y}$ of $\mathrm{C}_{G}(y)$, such that $x y=z$ and $P_{z} \leq P_{x} \cap P_{y}$.

Proof. See the proof of [1] Lemma 87.9.

Proof of Theorem 2, $Z_{p^{\prime}}^{S_{n}}$ is an algebra by Corollary 5 of [14. Now let $\left(a_{1}, \ldots, a_{n}\right)$ and $\left(b_{1}, \ldots, b_{n}\right)$ be cycle types of $p$-near-regular $p^{\prime}$-partitions $\sigma$ and $\tau$. We have to show that $C_{\pi} \not \subset \operatorname{supp} C_{\sigma}^{+} C_{\tau}^{+}$for $p^{\prime}$-classes $C_{\pi}$, which are not $p$-near-regular.

Let $\left(c_{1}, \ldots, c_{n}\right)$ be the cycle type of $\pi$. If $c_{1} \geq p$ we can apply the Brauer-homomorphism and get

$$
\operatorname{Br}_{\langle(1, \ldots, p)\rangle}\left(C_{\pi}^{+}\right)=C_{\pi^{\prime}}^{+} \neq 0,
$$

where $\pi^{\prime}$ has cycle type $\left(c_{1}-p, c_{2}, \ldots, c_{n-p}\right)$. Thus we get $\left.c_{\sigma \tau \pi}\right|_{S_{n}}=\left.c_{\sigma^{\prime} \tau^{\prime} \pi^{\prime}}\right|_{S_{n-p}}$, where $C_{\sigma^{\prime}}^{+}=\operatorname{Br}_{\langle(1, \ldots, p)\rangle}\left(C_{\sigma}^{+}\right)$and analogously for $C_{\tau^{\prime}}^{+}$. Moreover, $C_{\sigma^{\prime}}$ and $C_{\tau^{\prime}}$ are $p$-near-regular $p^{\prime}$-classes. Therefore we can assume that $c_{1}<p$.

Now let $z \in C_{\pi}$ and let $x \in C_{\sigma}, y \in C_{\tau}$ such that $x y=z$. It is well known that

$$
\mathrm{C}_{S_{n}}(x) \cong S_{a_{1}} \times\left(\mathbb{Z}_{2} \succ S_{a_{2}}\right) \times \ldots \times\left(\mathbb{Z}_{n} \succ S_{a_{n}}\right)
$$

and $\left|\mathbb{Z}_{k} \succ S_{k}\right|=a_{k} ! \cdot k^{a_{k}}$ ([17], 3.2.13). As $a_{k}<p$ for $k \geq 2$ and $a_{k}=0$ for $p \mid k$ we obtain that $p$ divides $\left|\mathrm{C}_{S_{n}}(x)\right|$ if and only if $a_{1} \geq p$.

Hence the Sylow- $p$-subgroup of $\mathrm{C}_{S_{n}}(x)$ is a subgroup of the subgroup isomorphic to $S_{a_{1}}$. This subgroup is the symmetric group on the set of numbers which are fixed by $x$. The same is true for $y$. Now let $P_{x}, P_{y}$ be Sylow-p-subgroups of $\mathrm{C}_{S_{n}}(x)$, $\mathrm{C}_{S_{n}}(y)$, respectively and let $P:=P_{x} \cap P_{y}$. Then all numbers which are moved by an element of $P$ are fixed by $x$ and $y$ and therefore are fixed by $z$. But $c_{1}<p$, so $z$ fixes less than $p$ numbers, i.e. no permutation of the fixed points of $z$ has order $p$. Thus we obtain $P_{x} \cap P_{y}=\langle 1\rangle$.

If $P_{z}$ is a Sylow-p-subgroup of $\mathrm{C}_{S_{n}}(z)$, then $\left|P_{z}\right|>1$ because there is an $i \geq 2$ such that $c_{i} \geq p$. Thus we obtain $P_{z} \not \subset P_{x} \cap P_{y}$ for all $x \in C_{\sigma}, y \in C_{\tau}$ with $x y=z$. By Lemma 13 we get $C_{\pi} \not \subset \operatorname{supp}\left(C_{\sigma}^{+} C_{\tau}^{+}\right)$.

Table 1 shows the dimension of $U_{p}:=Z_{p^{\prime}}^{S_{n}} \cap Z_{p-n r e g}^{S_{n}}$ for $p=2,3$ in comparison with the dimensions of $Z\left(\mathbb{F}_{p} S_{n}\right)$ and $Z_{p^{\prime}}^{S_{n}}$.

The following remark is a special case of a more general theorem of Osima already mentioned above ([15, Theorem 4.1]).

Remark 14. $Z_{p^{\prime}}^{S_{n}} \cap Z_{p-r e g}^{S_{n}}$ is an algebra.

For the rest of this section we fix the following notation: 


\begin{tabular}{r|r|r|r|r|r}
$n$ & $\operatorname{dim} Z\left(\mathbb{F}_{p} S_{n}\right)$ & $\operatorname{dim} Z_{2^{\prime}}^{S_{n}}$ & $\operatorname{dim} U_{2}$ & $\operatorname{dim} Z_{3^{\prime}}^{S_{n}}$ & $\operatorname{dim} U_{3}$ \\
\hline 10 & 42 & 10 & 7 & 22 & 18 \\
20 & 627 & 64 & 30 & 202 & 129 \\
30 & 5604 & 296 & 95 & 1225 & 622 \\
40 & 37338 & 1113 & 260 & 5834 & 2405 \\
50 & 204226 & 3658 & 632 & 23603 & 8008
\end{tabular}

TABLE 1.

Notation 15. By $\sigma=\left(a_{1}, \ldots, a_{n}\right)$ we denote the cycle type of a $p^{\prime}$-partition with $a_{i} \geq p$ for an $i \geq 2, a_{j}<p$ for $j<i$. $C_{\sigma}$ denotes the conjugacy class of elements of cycle type $\sigma$ in $S_{n}$. Thus the $p^{\prime}$-partition $\sigma$ is not $p$-regular, but elements in $C_{\sigma}$ fix at most $p-1$ symbols in $\{1, \ldots, n\}$.

The idea of the proof of Theorem 3 is the following: If the support of an idempotent $e$ contains $C_{\sigma}$, then the support also contains $\operatorname{supp}\left(C_{\sigma}^{+}\right)^{p}$. The support of $\left(C_{\sigma}^{+}\right)^{p}$ can contain classes $C_{\mu}$, where $\mu$ is not $p$-regular. But if $\left(b_{1}, \ldots, b_{n}\right)$ is the cycle type of such a class $C_{\mu}$ and if $b_{k} \geq p$, then $k>i$, i.e. the position where the 'irregularity' occurs, grows. So a class $C_{\sigma}$ in the support of $e$ with a 'minimal position of irregularity' is not contained in the support of $e^{p}=e$, a contradiction.

For the proof of Theorem 3 we first collect the necessary information about $C_{\sigma}$ in several lemmas. We start with a lemma about the centralizers of an element of cycle type $\sigma$.

Lemma 16. Let $x \in C_{\sigma}$ and let $y \in \mathrm{C}_{S_{n}}(x)$ have order $p$. Then $y$ is a product of $i$ or more commuting p-cycles.

Proof. As $y$ centralizes $x$, it permutes the orbits of $x$ on $\{1, \ldots, n\}$. Let $O$ be one orbit of $x$ which is not fixed by $y$. Then $O, y O, \ldots, y^{p-1} O$ are distinct orbits of $x$. Thus the cycle decomposition of $x$ consists of at least $p$ cycles of length $|O|$. Our choice of $x$ gives $|O| \geq i$, and $y$ is a product of at least $|O|$ commuting $p$-cycles.

Corollary 17. Let $\sigma$ be as in Notation 15, let $C_{\mu} \subset S_{n}$ be a $p^{\prime}$-conjugacy class and let $\lambda=\left(c_{1}, \ldots, c_{n}\right)$ be the cycle type of a $p^{\prime}$-partition with $C_{\lambda} \subset \operatorname{supp}\left(C_{\mu}^{+} C_{\sigma}^{+}\right)$. Then $c_{j}<p$ for $2 \leq j<i$.

Proof. Let $x \in C_{\mu}, y \in C_{\sigma}$ and $z \in C_{\lambda}$. Suppose that $z$ contains at least $p$ commuting $j_{0}$-cycles in its cycle decomposition, where $j_{0}<i$. Then an element permuting $p$ of the orbits of $z$ on $\{1, \ldots, n\}$ of length $j_{0}$ is an element in $C_{S_{n}}(z)$, and its cycle decomposition consists of $j_{0}$ commuting $p$-cycles. Thus every Sylow$p$-subgroup $P_{z}$ of $\mathrm{C}_{S_{n}}(z)$ contains an element of this cycle type. But $\mathrm{C}_{S_{n}}(x)$ does not contain an element of such a cycle type according to Lemma 16. Hence if $P_{x}$, $P_{y}$ are Sylow- $p$-subgroups of $\mathrm{C}_{S_{n}}(x), \mathrm{C}_{S_{n}}(y)$, respectively, then $P_{z} \not \subset P_{x} \cap P_{y}$ and by Lemma 13 we obtain $C_{\lambda} \not \subset \operatorname{supp}\left(C_{\mu}^{+} C_{\lambda}^{+}\right)$.

We remark that we just proved that a defect group of $C_{\lambda}$ is not contained in a defect group of $C_{\mu}$ up to conjugacy, so Corollary 17 also follows by the theorem of Osima mentioned above ([15, Theorem 4.1]).

Lemma 18. Let $\pi$ be a product of $i$ commuting p-cycles, where $i$ is coprime to $p$. Then all $p^{\prime}$-elements of the same cycle type contained in $\mathrm{C}_{S_{p \cdot i}}(\pi)$ are conjugate in $\mathrm{C}_{S_{p \cdot i}}(\pi)$. 
Proof. $\mathrm{C}_{S_{p \cdot i}}(\pi) \cong \mathbb{Z}_{p} \prec S_{i}$ (see $\left.(*)\right)$ is a semidirect product of a $p$-group (generated by the cycles of $\pi$ ) and a copy of $S_{i}$. Using the explicit description of the conjugacy classes of $\mathbb{Z}_{p} 2 S_{i}$ in [9, 3.2, 3.13] we can deduce that every $p^{\prime}$-element of $\mathrm{C}_{S_{p \cdot i}}(\pi)$ is conjugate in $\mathrm{C}_{S_{p . i}}(\pi)$ to an element of the copy of $S_{i}$. Therefore the projection $\mathrm{C}_{S_{p \cdot i}}(\pi) \longrightarrow S_{i}$ induces a bijection between the $p^{\prime}$-classes of the two groups. Due to [7, $4.1 .18,4.2 .17]$ this bijection maps a $p^{\prime}$-element of cycle type $\left(p \lambda_{1}, \ldots, p \lambda_{i}\right)$ to an element of cycle type $\left(\lambda_{1}, \ldots, \lambda_{i}\right)$. Thus we obtain our result.

Corollary 19. We keep the notation of Lemma 18, Let $g \in \mathrm{C}_{S_{p \cdot i}}(\pi)$ be a $p^{\prime}$ element and let $\chi \in \operatorname{Irr} \mathrm{C}_{S_{p \cdot i}}(\pi)$. Then $\chi(g) \in \mathbb{Z}$.

Proof. Let $m \in \mathbb{N}$ with $\operatorname{gcd}(m$, ord $g)=1$. By Lemma 18 the elements $g$ and $g^{m}$ are conjugate in $\mathrm{C}_{S_{p \cdot i}}(\pi)$, thus we obtain $\chi(g) \in \mathbb{Z}$ for $\chi \in \operatorname{Irr} \mathrm{C}_{S_{p \cdot i}}(\pi)$ using $[5$, V.13.7.b)] and [4, 6.3b), 6.4a)].

Remark 20. With the notation of Lemma 18 the vector space $Z_{p^{\prime}}^{\mathrm{C}_{S_{p \cdot i}}(\pi)}$ is an algebra.

Proof. $Z_{p^{\prime}}^{S_{p \cdot i}}$ is an algebra by Corollary 5 of [14], and the Brauer homomorphism

$$
\operatorname{Br}_{\langle\pi\rangle}: Z\left(\mathbb{F}_{p} S_{p \cdot i}\right) \longrightarrow Z\left(\mathbb{F}_{p} \mathrm{C}_{S_{p \cdot i}}(\pi)\right)
$$

is a projection. By Lemma 18 this projection is surjective: If $C$ is a $p^{\prime}$-class of $S_{p \cdot i}$, then $C \cap C_{S_{p \cdot i}}(\pi)=\emptyset$ or $C \cap C_{S_{p \cdot i}}(\pi)$ is a $p^{\prime}$-class of $C_{S_{p \cdot i}}(\pi)$.

Lemma 21. Let $\pi$ and $i$ be as in Lemma 18, Let $C \subset \mathrm{C}_{S_{p \cdot i}}(\pi)$ be the conjugacy class of elements of cycle type $\left(c_{1}, \ldots, c_{p i}\right)$, where $c_{i}=p$ and $c_{j}=0$ for $j \neq i$. Then $\left(C^{+}\right)^{2}=0$ in $\mathbb{F}_{p} \mathrm{C}_{S_{p \cdot i}}(\pi)$.

Proof. $\operatorname{supp}\left(C^{+}\right)^{2}$ consists of $p^{\prime}$-elements according to Remark 20. Now let $D \subset$ $\mathrm{C}_{S_{p \cdot i}}(\pi)$ be a $p^{\prime}$-conjugacy class. According to [4, 4.6, the coefficient $a_{C C D}$ of $D^{+}$ in $\left(C^{+}\right)^{2} \in \mathbb{Z} \mathrm{C}_{S_{p . i}}(\pi)$ is

$$
a_{C C D}=\frac{|C|^{2}}{\left|\mathrm{C}_{S_{p \cdot i}}(\pi)\right|} \sum_{\chi \in \operatorname{Irr}_{C_{S_{p}, i}}(\pi)} \chi^{2}(g) \cdot \frac{\chi\left(h^{-1}\right)}{\chi(1)},
$$

where $g \in C$ and $h \in D$. By Corollary 19 we know that $\chi(g)$ and $\chi\left(h^{-1}\right)$ are integers. Using [9], 3.9, we obtain

$$
\frac{|C|^{2}}{\left|\mathrm{C}_{S_{p \cdot i}}(\pi)\right|}=\frac{\left(p^{i-1} \cdot(i-1) !\right)^{2}}{p^{i} \cdot i !}=\frac{p^{i-2}(i-1) !}{i} .
$$

The group $\mathrm{C}_{S_{p \cdot i}}(\pi) \cong\left(\mathbb{Z}_{p}\right)^{i} \rtimes S_{i}$ contains an abelian normal subgroup of order $p^{i}$, so by a theorem of Ito $([4,19.9)$ we get

$$
\chi(1) \mid\left[\mathrm{C}_{S_{p \cdot i}}(\pi):\left(\mathbb{Z}_{p}\right)^{i}\right]=\frac{p^{i} \cdot i !}{p^{i}}=i !
$$

As $p \nmid i$ this provides $a_{C C D} \equiv 0 \bmod p$ for $i>2$. For $i=2$ the centralizer of $\pi$ is a group of order $2 p^{2}$. Therefore the product of two elements of $C$ is an element of the Sylow-p-subgroup of $\mathrm{C}_{S_{p \cdot i}}(\pi)$, i.e. $C \not \subset \operatorname{supp}\left(C^{+}\right)^{2}$. As $p|| C \mid$ provides $1 \notin \operatorname{supp}\left(C^{+}\right)^{2}$, we obtain $a_{C C D} \equiv 0 \bmod p$ for the case $i=2$, because $\{1\}$ and $C$ are the only $p^{\prime}$-classes of $\mathrm{C}_{S_{p \cdot i}}(\pi)$ in this case. 
We remark that the fact that $C^{+}$is nilpotent can already be deduced from [15, 4.7].

Lemma 22. Let $\sigma, i$ be as in Notation 15, and let $\mu=\left(b_{1}, \ldots, b_{n}\right)$ be the cycle type of a $p^{\prime}$-partition with $b_{i} \geq p$. Then $C_{\mu} \not \subset \operatorname{supp}\left(C_{\sigma}^{+}\right)^{p}$.

Proof. There are elements $x \in C_{\sigma}$ and $y \in C_{\mu}$ such that

$$
\pi=(1,2, \ldots, p)(p+1, \ldots, 2 p) \ldots((i-1) p+1, \ldots, i p)
$$

is an element of $\mathrm{C}_{S_{n}}(x)$ and of $\mathrm{C}_{S_{n}}(y)$. We apply the Brauer-homomorphism

$$
\operatorname{Br}_{\langle\pi\rangle}: Z\left(\mathbb{F}_{p} S_{n}\right) \longrightarrow Z\left(\mathbb{F}_{p} \mathrm{C}_{S_{n}}(\pi)\right)
$$

and get

$$
C_{\mu} \subset \operatorname{supp}\left(C_{\sigma}^{+}\right)^{p} \Longleftrightarrow \operatorname{supp} \operatorname{Br}_{\langle\pi\rangle}\left(C_{\mu}\right)^{+} \subset \operatorname{supp}\left(\operatorname{Br}_{\langle\pi\rangle}\left(C_{\sigma}^{+}\right)\right)^{p},
$$

as $\operatorname{Br}_{\langle\pi\rangle}\left(C_{\mu}\right)^{+} \neq 0$.

The coefficient $a_{\mu}$ of $C_{\mu}^{+}$in $\left(C_{\sigma}^{+}\right)^{p} \in \mathbb{Z} S_{n}$ is the number of solutions $\left(g_{1}, \ldots, g_{p}\right)$ of the equation

$$
g_{1} \cdot \ldots \cdot g_{p}=y,
$$

where $g_{i} \in C_{\sigma} \cap \mathrm{C}_{S_{n}}(\pi)$. Using the decomposition $\mathrm{C}_{S_{n}}(\pi) \cong \mathrm{C}_{S_{p . i}}(\pi) \times S_{n-i p}$ we can write $g_{t}=g_{t, 1} g_{t, 2}$ and $y=y_{1} y_{2}$ with $g_{t, 1}, y_{1} \in \mathrm{C}_{S_{p . i}}(\pi)$ and $g_{t, 2}, y_{2} \in S_{n-i p}$ and obtain equations

$$
g_{1,1} g_{2,1} \ldots g_{p, 1}=y_{1}, \quad g_{1,2} \ldots g_{p, 2}=y_{2} .
$$

If $r_{1}, r_{2}$ denote the number of solutions of the first and second equation, respectively, then $a_{\mu}=r_{1} \cdot r_{2}$. We show that $r_{1} \equiv 0 \bmod p$.

According to Lemma 18 there is exactly one conjugacy class $C \subset \mathrm{C}_{S_{p i}}(\pi)$ of elements of cycle type $\left(c_{1}, \ldots, c_{p i}\right)$ with $c_{i} \neq 0$ and $c_{j}=0$ for $j \neq i$, and in fact $c_{i}=p$ for this class. Let $g_{t}=g_{t, 1} g_{t, 2}$ be a decomposition of an element $g_{t} \in C_{\sigma} \cap \mathrm{C}_{S_{n}}(\pi)$. We prove that $g_{t, 1} \in C$ and that for every element $g_{t, 1} \in C$ there exists an element $g_{t, 2} \in S_{n-i p}$ such that $g_{t, 1} g_{t, 2} \in C_{\sigma} \cap \mathrm{C}_{S_{n}}(\pi)$. Thus we have to count the number of solutions $\left(g_{1,1}, \ldots, g_{p, 1}\right)$ of the first equation, where $g_{t, 1} \in C$ for all $t$. Then we prove that $y_{1} \in C$ as well and that therefore $r_{1}$ is the coefficient of $C^{+}$in $\left(C^{+}\right)^{p}$.

All $p^{\prime}$-elements in $\mathrm{C}_{S_{p i}}(\pi) \backslash\{(1)\}$ have a cycle type of the form $\left(p d_{1}, \ldots, p d_{i}\right)$. As $a_{j}<p$ for $1 \leq j<i$ we obtain $g_{t, 1} \in C$ for all $g_{t}=g_{t, 1} g_{t, 2} \in \mathrm{C}_{S_{n}}(\pi) \cap C_{\sigma}$ and for every $g_{t, 1} \in C$ there exists a $g_{t, 2} \in S_{n-i p}$ such that $g_{t}=g_{t, 1} g_{t, 2} \in \mathrm{C}_{S_{n}}(\pi) \cap C_{\sigma}$.

Now let $\lambda=\left(b_{1}, \ldots, b_{n}\right)$ be the cycle type of a $p^{\prime}$-partition with $C_{\lambda} \subset \operatorname{supp}\left(C_{\sigma}^{+}\right)^{k}$. Using induction and Corollary 17 we see that $b_{j}<p$ for all $j<i$. This also provides $y_{1} \in C$ for all $y=y_{1} y_{2} \in \mathrm{C}_{S_{n}}(\pi) \cap C_{\mu}$. Therefore $r_{1}$ is the coefficient of $C^{+}$in $\left(C^{+}\right)^{p} \in Z\left(\mathbb{F}_{p} \mathrm{C}_{S_{p \cdot i}}(\pi)\right)$. But by Lemma 21 we have $\left(C^{+}\right)^{2}=0$ and thus $\left(C^{+}\right)^{p}=0$, i.e. $r_{1} \equiv 0 \bmod p$.

Corollary 23. Let $\sigma, i$ be as in Notation 15, and let $\mu=\left(b_{1}, \ldots, b_{n}\right)$ be the cycle type of a $p^{\prime}$-partition with $C_{\mu} \subset \operatorname{supp}\left(C_{\sigma}^{+}\right)^{p}$. Then $b_{j}<p$ for $1 \leq j \leq i$.

Proof. For $1 \leq j<i$ the statement $b_{j}<p$ follows by induction and Corollary 17 . Lemma 22 provides $b_{i}<p$.

Proof of Theorem 3. We consider a minimal counterexample in the following sense: For a given prime $p$ let $n$ be minimal such that a primitive central idempotent $e \in \mathbb{F}_{p} S_{n}$ exists with $e \notin Z_{p^{\prime}}^{S_{n}} \cap Z_{p-n r e g}^{S_{n}}$. According to a theorem of Osima $([8], 7.4)$ we know that $e \in Z_{p^{\prime}}^{S_{n}}$, therefore there is a $p^{\prime}$-partition $\sigma$ of cycle type $\left(a_{1}, \ldots, a_{n}\right)$ 
with $a_{i} \geq p$ for some $i \geq 2$, such that $C_{\sigma} \subset \operatorname{supp} e$. Now we choose $\sigma$ to be the partition, where $i \geq 2$ is minimal with $a_{i} \geq p$ under all partitions with $C_{\sigma} \subset \operatorname{supp} e$.

Let $e=\sum_{C_{\tau} \subset \operatorname{supp} e} a_{\tau} C_{\tau}^{+}$. Then

$$
e=e^{p}=\sum_{C_{\tau} \subset \operatorname{supp} e} a_{\tau}^{p}\left(C_{\tau}^{+}\right)^{p}=\sum_{C_{\tau} \subset \operatorname{supp} e} a_{\tau}\left(C_{\tau}^{+}\right)^{p} .
$$

For conjugacy class sums $C_{\tau}^{+} \in Z_{p^{\prime}}^{S_{n}} \cap Z_{p-n r e g}^{S_{n}}$ we know $\left(C_{\tau}^{+}\right)^{p} \in Z_{p^{\prime}}^{S_{n}} \cap Z_{p-n r e g}^{S_{n}}$ by Theorem 2, thus $C_{\sigma} \not \subset \operatorname{supp}\left(C_{\tau}^{+}\right)^{p}$ for these $\tau$. Now let $\left(b_{1}, \ldots, b_{n}\right)$ be the cycle type of a partition $\tau$ with $C_{\tau} \subset \operatorname{supp} e$ and $b_{j} \geq p$ for some $j \geq 2$. Using Theorem 1 and the minimality of $n$ we get that $b_{1}<p$ for all these $\tau$. Now let $j$ be minimal with $b_{j} \geq p$ for the given $\tau$. If $j \geq i$, then $C_{\sigma} \not \subset \operatorname{supp}\left(C_{\tau}^{+}\right)^{p}$ according to Corollary 23 As we chose $i$ to be minimal there is no $\tau$ such that $C_{\tau} \subset \operatorname{supp} e$ and $b_{j} \geq p$ for a $j$ with $2 \leq j<i$. Hence we obtain

$$
C_{\sigma} \not \subset \operatorname{supp} e^{p}=\operatorname{supp} e,
$$

a contradiction.

Now we want to use the theorems we proved to speed up the algorithm described in Algorithm 7 for the computation of the primitive central idempotents of finite group rings of symmetric and alternating groups. For symmetric groups the number $r$ of primitive central idempotents of $\mathbb{F}_{p} S_{n}$ can be computed using Nakayamas Conjecture, [7, 6.1.21. To compute the primitive central idempotents of $\mathbb{F}_{p} S_{n}$ we use Algorithm 7 with the following changes:

- We compute the $p$-near-regular $p^{\prime}$-conjguacy classes in step 1) of Algorithm 7

- We subsume steps 2), 3) and 4) of Algorithm 7 in a loop and check if the basis computed in 4) already has $r$ elements. If this is the case we do not need to compute further idempotents and we can continue with step 5 .

- For step 2) and step 5) of Algorithm[7it is necessary to compute the product of class sums in $\mathbb{F}_{p} S_{n}$. We compute the class multiplication coefficients over the field $\mathbb{C}$ of complex numbers according to [4, Theorem 4.6, using a program written in SYMMETRICA ([10]) provided by A. Kohnert and then reduce them modulo $p$. As we are multiplying class sums of $p$-nearregular $p^{\prime}$-classes we only have to compute coefficients $c_{i j k}$ of $p$-near regular $p^{\prime}$-classes $C_{k}$ according to Theorem 2. We also use Corollary 12 to reduce the number of coefficients we have to compute.

- We store the products of class sums because they usually occur several times during the computation.

Considering the changes described above we see that the best situation occurs for $p=2$, because in this case the numbers of $p^{\prime}$-classes and of $p$-near-regular classes both are much smaller than for all other primes (see Table 1). But there are even more possibilities to speed up the program for $p=2$. The step of Algorithm 7 consuming the most time is step 5), because the idempotents $f_{i}$ are sums of many class sums and it takes a long time to compute a product $f_{i} \cdot f_{j}$. The philosophy for $p=2$ is to compute only squares of class sums if possible. So for $p=2$ we also made the following changes of Algorithm 7 , 
- It turned out that we get the right number of idempotents using 1 and the powers of the class sums $C_{3}^{+}, C_{7}^{+}, \ldots, C_{4 \cdot(r-2)+3}^{+}$. We didn't prove that the powers of these class sums always generate the vector space spanned by the primitive central idempotents, but the program will stop if it doesn't find enough linear independent idempotents.

- In step 2) we compute $\left(C_{i}^{+}\right)^{2^{j}}$ until $\left(C_{i}^{+}\right)^{2^{k}}=\left(C_{i}^{+}\right)^{2^{k+1}}$ building only squares of class sums. Then $f_{i}:=\left(C_{i}^{+}\right)^{2^{k}}$ is the idempotent occuring in step 3). We keep in mind that $f_{i}$ "comes from" $C_{i}$ for step 5 ).

- We store all class multiplication coefficients, because we can use them for bigger symmetric groups according to Corollary 12 .

- The products $f_{i} \cdot f_{j}$ occuring in step 5) are computed in the following way: We kept in mind that $f_{i}$ came from $C_{i}$ and $f_{j}$ came from $C_{j}$. So instead of multiplying $f_{i}$ and $f_{j}$ directly-both are usually large sums of class sums - we multiply $C_{i}^{+}$and $C_{j}^{+}$and compute $\left(C_{i}^{+} C_{j}^{+}\right)^{2^{j}}$ until $\left(C_{i}^{+} C_{j}^{+}\right)^{2^{k}}=$ $\left(C_{i}^{+} C_{j}^{+}\right)^{2^{k+1}}$. Then $f_{i} \cdot f_{j}=\left(C_{i}^{+} C_{j}^{+}\right)^{2^{k}}$. The advantage of this procedure is that we only have to compute squares of class sums and no mixed products except $C_{i}^{+} C_{j}^{+}$. We store all squares we compute, because they usually occur several times.

If we replace an idempotent $f_{i}$ by $f_{i} \cdot f_{j}$ or by a sum of idempotents, then we have to keep in mind that our new idempotent comes from $C_{i}^{+} \cdot C_{j}^{+}$ or from a sum of class sums. Here we get a delicate problem: After several steps of the loop in step 5) our idempotents are powers of expressions like $\left(C_{i}^{+}\right)^{2} \cdot C_{j}^{+}+\left(C_{k}^{+}\right)^{3} \cdot C_{m}^{+}+C_{k}^{+} \cdot\left(C_{m}^{+}\right)^{2}+\ldots$. In every step the expressions become longer and the multiplying of two such expressions takes more and more time. Therefore we have to keep these expressions simple: The idempotent generated by $\left(C_{i}^{+}\right)^{2} \cdot C_{j}$ is also generated by $C_{i}^{+} \cdot C_{j}$ so we have to filter out powers in our expressions. If we do that we will see that the idempotents generated by the summands $\left(C_{k}^{+}\right)^{3} \cdot C_{m}^{+}$and $C_{k}^{+} \cdot\left(C_{m}^{+}\right)^{2}$ in the expression above are the same, so we can delete those summands from our expression. Thus for every idempotent $f_{i}$ we store such an expression, and this expression has to be updated and simplified whenever we replace an idempotent $f_{i}$ by a product or a sum of idempotents.

These changes allowed us to compute the primitive central idempotents of $\mathbb{F}_{2} S_{n}$ for $n \leq 50$. The results can be seen in the Appendix. For our computations we used a dual core computer with two Opteron $2651.8 \mathrm{GHz}$ processors. Approximately the time needed to compute the idempotents of $S_{n+2}$ using the results of the computation for $S_{n}$ is twice the time needed for the computation of the idempotents for $S_{n}$. So it takes about $7 \mathrm{~s}$ to carry out the computation for $S_{20}, 4 \mathrm{~m}$ for $S_{30}, 3 \mathrm{~h} 31 \mathrm{~m}$ for $S_{40}$ and $10 \mathrm{~d} 7 \mathrm{~h} 14 \mathrm{~m}$ for $S_{50}$ using the results for $S_{28}, S_{38}$ and $S_{48}$, respectively.

The necessary information about the primitive central idempotents of the group rings $\mathbb{F}_{2} A_{n}$ can be found in corollaries 4 and 5 of [3]: If $n$ is not of the form $n=\frac{m(m+1)}{2}$ with an integer $m$, then the primitive central idempotents of $\mathbb{F}_{2} S_{n}$ are also the primitive central idempotents of $\mathbb{F}_{2^{k}} A_{n}$. If $n=\frac{m(m+1)}{2}$, then this is also true for all idempotents of $\mathbb{F}_{2} S_{n}$ except for one. In this case $e=C^{+}$is a primitive central idempotent of $\mathbb{F}_{2} S_{n}$ according to Theorem 3 of [3], where $C$ is the conjugacy class of elements corresponding to the partition $(2 m-1,2 m-5,2 m-9, \ldots)$ of $n$. 
This idempotent $e$ splits in a sum of two primitive central idempotents of $\mathbb{F}_{2^{k}} A_{n}$. As the class $C$ splits in two conjugacy classes $C_{-}$and $C_{+}$of $A_{n}$ we can compute the remaining primitive central idempotents of $\mathbb{F}_{2^{k}} A_{n}$ by computing the powers of the class sums $C_{-}^{+}$and $C_{+}^{+}$. For alternating groups the field $\mathbb{F}_{2}$ is not always a splitting field, but at least $\mathbb{F}_{4}$ is a splitting field for $A_{n}$ according to Corollary $\mathrm{B}$ of [13. Thus it may happen that the two idempotents are not elements of $\mathbb{F}_{2} A_{n}$, but they are elements of $\mathbb{F}_{4} A_{n}$. The results show that our Theorems 1,2,3 are not true for alternating groups.

\section{ACKNOWLEDGEMENTS}

The author thanks A. Kerber and W. Müller for fruitful discussions about this topic, A. Kohnert for providing programs computing the class multiplication coefficients of symmetric and alternating groups, A. Konovalov for help with a lot of questions concerning GAP, and the referee for a lot of helpful comments and suggestions.

\section{APPENDIX}

Here our notation is slightly different to the notation in the rest of the article: If $\mu=2^{\alpha_{2}}, \ldots, n^{\alpha_{n}}$ is a partition we write $\overline{2^{\alpha_{2}}, \ldots, n^{\alpha_{n}}}$ for the class sum $C_{\mu}^{+} \in \mathbb{F}_{2} S_{m}$, where $m \geq W(\mu)$. According to Theorem 1 one can easily deduce the primitive central idempotents of $\mathbb{F}_{2} S_{n}$ for $n<49$ from the primitive central idempotents of $\mathbb{F}_{2} S_{50}$ and $\mathbb{F}_{2} S_{49}$. To simplify that task we added tokens of the form $\left.\right|_{16}$ to indicate where the primitive central idempotent of $\mathbb{F}_{2} S_{16}$ ends.

Primitive central idempotents of $\mathbb{F}_{2} S_{n}$ for $n$ odd and $n \leq 49$ :

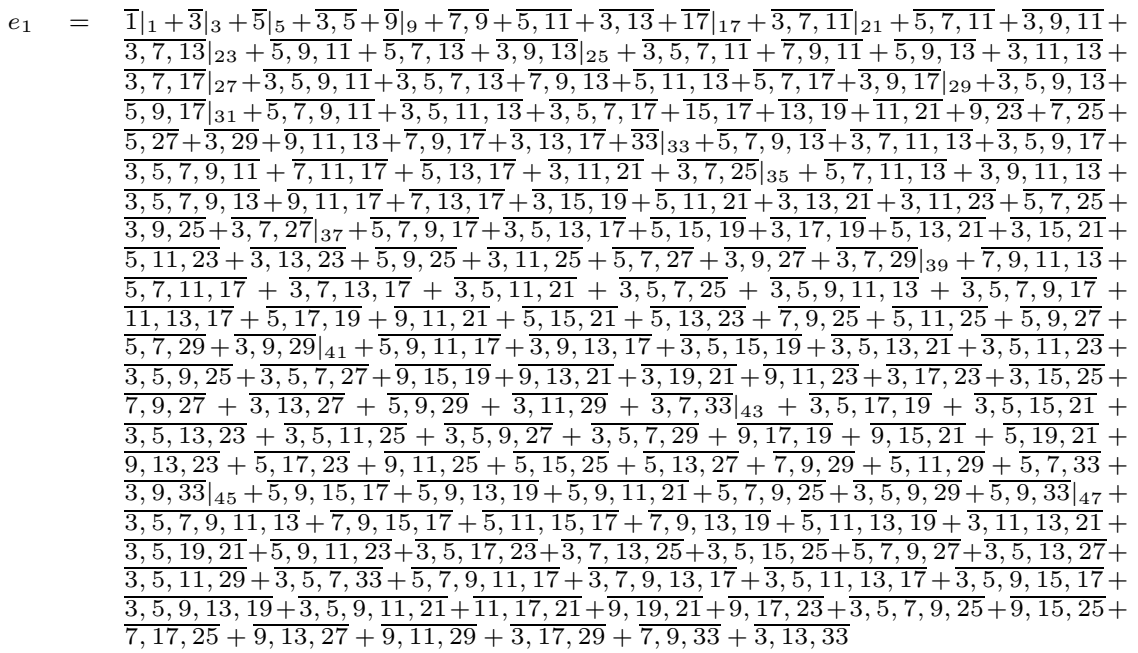


$e_{2}=\left.\overline{3}\right|_{3}+\left.\overline{5}\right|_{5}+\overline{3,5}+\left.\overline{9}\right|_{9}+\left.\overline{5,9}\right|_{15}+\overline{3,13}+\overline{3,5,9}+\left.\overline{17}\right|_{17}+\overline{7,11}+\overline{5,13}+\overline{3,7,9}+$ $\left.\overline{3,5,11}\right|_{19}+\overline{9,11}+\overline{7,13}+\overline{5,7,9}+\overline{3,7,11}+\left.\overline{3,5,13}\right|_{21}+\overline{5,17}+\overline{5,7,11}+\overline{3,9,11}+$ $\left.\overline{3,7,13}\right|_{23}+\overline{3,5,7,9}+\overline{11,13}+\overline{7,17}+\overline{5,7,13}+\left.\overline{3,5,17}\right|_{25}+\overline{3,5,7,11}+\overline{9,17}+\overline{7,9,11}+$ $\overline{5,9,13}+\overline{3,11,13}+\left.\overline{3,7,17}\right|_{27}+\overline{3,5,9,11}+\overline{3,5,7,13}+\overline{7,9,13}+\overline{5,11,13}+\overline{5,7,17}+$ $\left.\overline{3,9,17}\right|_{29}+\overline{3,5,9,13}+\left.\overline{5,9,17}\right|_{31}+\overline{3,7,9,13}+\overline{3,5,7,17}+\overline{11,21}+\overline{7,25}+\overline{3,29}+$ $\overline{9,11,13}+\overline{5,11,17}+\left.\overline{33}\right|_{33}+\overline{5,7,9,13}+\overline{3,7,11,13}+\overline{3,5,9,17}+\overline{15,19}+\overline{13,21}+$ $\overline{11,23}+\overline{9,25}+\overline{7,27}+\overline{5,29}+\overline{3,5,7,9,11}+\overline{7,11,17}+\overline{5,13,17}+\overline{3,15,17}+\overline{3,13,19}+$ $\overline{3,9,23}+\left.\overline{3,5,27}\right|_{35}+\overline{5,7,11,13}+\overline{3,9,11,13}+\overline{17,19}+\overline{15,21}+\overline{13,23}+\overline{11,25}+\overline{9,27}+$ $\overline{7,29}+\overline{3,5,7,9,13}+\overline{9,11,17}+\overline{7,13,17}+\overline{5,15,17}+\overline{5,13,19}+\overline{3,15,19}+\overline{3,13,21}+$ $\overline{5,9,23}+\overline{3,11,23}+\overline{3,9,25}+\overline{3,7,27}+\left.\overline{3,5,29}\right|_{37}+\overline{5,7,9,17}+\overline{3,5,13,17}+\overline{5,33}+$ $\overline{5,15,19}+\overline{3,17,19}+\overline{5,13,21}+\overline{3,15,21}+\overline{5,11,23}+\overline{3,13,23}+\overline{5,9,25}+\overline{3,11,25}+$ $\overline{5,7,27}+\overline{3,9,27}+\left.\overline{3,7,29}\right|_{39}+\overline{7,9,11,13}+\overline{5,7,11,17}+\overline{3,7,13,17}+\overline{3,5,15,17}+$ $\overline{3,5,13,19}+\overline{19,21}+\overline{3,5,9,23}+\overline{17,23}+\overline{15,25}+\overline{13,27}+\overline{11,29}+\overline{7,33}+\overline{3,5,9,11,13}+$ $\overline{3,5,7,9,17}+\overline{11,13,17}+\overline{9,15,17}+\overline{9,13,19}+\overline{5,17,19}+\overline{5,15,21}+\overline{5,13,23}+\overline{5,11,25}+$ $\overline{5,7,29}+\left.\overline{3,5,33}\right|_{41}+\overline{5,9,11,17}+\overline{3,9,13,17}+\overline{3,5,15,19}+\overline{3,5,13,21}+\overline{3,5,11,23}+$ $\overline{3,5,9,25}+\overline{3,5,7,27}+\overline{9,33}+\overline{9,15,19}+\overline{9,13,21}+\overline{3,19,21}+\overline{9,11,23}+\overline{3,17,23}+$ $\overline{3,15,25}+\overline{7,9,27}+\overline{3,13,27}+\overline{5,9,29}+\overline{3,11,29}+\left.\overline{3,7,33}\right|_{43}+\overline{3,5,17,19}+\overline{3,5,15,21}+$ $\overline{3,5,13,23}+\overline{3,5,11,25}+\overline{3,5,9,27}+\overline{3,5,7,29}+\overline{9,17,19}+\overline{9,15,21}+\overline{5,19,21}+$ $\overline{9,13,23}+\overline{5,17,23}+\overline{9,11,25}+\overline{5,15,25}+\overline{5,13,27}+\overline{7,9,29}+\overline{5,11,29}+\overline{5,7,33}+$ $3,9,\left.33\right|_{45}+\overline{5,9,15,17}+5,9,13,19+5,9,11,21+5,7,9,25+3,5,9,29+5,9,\left.33\right|_{47}+$ $\overline{3,5,7,9,11,13}+\overline{3,13,15,17}+\overline{7,9,11,21}+\overline{3,5,19,21}+\overline{3,9,13,23}+\overline{3,5,17,23}+$ $\overline{5,7,11,25}+\overline{3,5,15,25}+\overline{23,25}+\overline{21,27}+\overline{3,7,9,29}+\overline{19,29}+\overline{3,5,7,33}+\overline{15,33}+$ $\overline{5,7,9,11,17}+\overline{3,7,9,13,17}+\overline{3,5,11}, 13,17+\overline{3,5,9,15,17}+\overline{3,5,9,13,19}+\overline{13,17,19}+$ $\overline{3,5,9,11,21}+\overline{9,19,21}+\overline{3,5,7,9,25}+\overline{9,15,25}+\overline{9,13,27}+\overline{5,17,27}+\overline{9,11,29}+\overline{5,11,33}$

$\left.\overline{5,9}\right|_{15}+\overline{7,9}+\overline{5,11}+\left.\overline{3,5,9}\right|_{17}+\overline{7,11}+\overline{5,13}+\overline{3,7,9}+\left.\overline{3,5,11}\right|_{19}+\overline{9,11}+\overline{7,13}+$ $\overline{5,7,9}+\overline{3,7,11}+\left.\overline{3,5,13}\right|_{21}+\overline{5,17}+\overline{5,7,11}+\overline{3,9,11}+\left.\overline{3,7,13}\right|_{23}+\overline{3,5,7,9}+\overline{11,13}+$ $\overline{7,17}+\overline{5,7,13}+\left.\overline{3,5,17}\right|_{25}+\overline{3,5,7,11}+\overline{9,17}+\overline{7,9,11}+\overline{5,9,13}+\overline{3,11,13}+\left.\overline{3,7,17}\right|_{27}+$ $\overline{3,5,9,11}+\overline{3,5,7,13}+\overline{7,9,13}+\overline{5,11,13}+\overline{5,7,17}+\left.\overline{3,9,17}\right|_{29}+\overline{3,5,9,13}+\left.\overline{5,9,17}\right|_{31}+$ $\overline{3,7,9,13}+\overline{3,5,7,17}+\overline{15,17}+\overline{13,19}+\overline{9,23}+\overline{5,27}+\overline{9,11,13}+\left.\overline{5,11,17}\right|_{33}+\overline{5,7,9,13}+$ $\overline{3,7,11,13}+\overline{3,5,9,17}+\overline{15,19}+\overline{13,21}+\overline{11,23}+\overline{9,25}+\overline{7,27}+\overline{5,29}+\overline{3,5,7,9,11}+$ $\overline{7,11,17}+\overline{5,13,17}+\overline{3,15,17}+\overline{3,13,19}+\overline{3,9,23}+\left.\overline{3,5,27}\right|_{35}+\overline{5,7,11,13}+\overline{3,9,11,13}+$ $\overline{17,19}+\overline{15,21}+\overline{13,23}+\overline{11,25}+\overline{9,27}+\overline{7,29}+\overline{3,5,7,9,13}+\overline{9,11,17}+\overline{7,13,17}+$ $\overline{5,15,17}+\overline{5,13,19}+\overline{3,15,19}+\overline{3,13,21}+\overline{5,9,23}+\overline{3,11,23}+\overline{3,9,25}+\overline{3,7,27}+$ $\left.\overline{3,5,29}\right|_{37}+\overline{5,7,9,17}+\overline{3,5,13,17}+\overline{5,33}+\overline{5,15,19}+\overline{3,17,19}+\overline{5,13,21}+\overline{3,15,21}+$ $\overline{5,11,23}+\overline{3,13,23}+\overline{5,9,25}+\overline{3,11,25}+\overline{5,7,27}+\overline{3,9,27}+\left.\overline{3,7,29}\right|_{39}+\overline{7,9,11,13}+$ $\overline{5,7,11,17}+\overline{3,7,13,17}+\overline{3,5,15,17}+\overline{3,5,13,19}+\overline{19,21}+\overline{3,5,9,23}+\overline{17,23}+\overline{15,25}+$ $\overline{13,27}+\overline{11,29}+\overline{7,33}+\overline{3,5,9,11,13}+\overline{3,5,7,9,17}+\overline{11,13,17}+\overline{9,15,17}+\overline{9,13,19}+$ $\overline{5,17,19}+5,15,21+\overline{5,13,23}+5,11,25+5,7,29+\left.\overline{3,5,33}\right|_{41}+\overline{5,9,11,17}+\overline{3,9,13,17}+$ $\overline{3,5,15,19}+\overline{3,5,13,21}+\overline{3,5,11,23}+\overline{3,5,9,25}+\overline{3,5,7,27}+\overline{9,33}+\overline{9,15,19}+\overline{9,13,21}+$ $\overline{3,19,21}+\overline{9,11,23}+\overline{3,17,23}+\overline{3,15,25}+\overline{7,9,27}+\overline{3,13,27}+\overline{5,9,29}+\overline{3,11,29}+$ $\left.\overline{3,7,33}\right|_{43}+\overline{3,5,17,19}+\overline{3,5,15,21}+\overline{3,5,13,23}+\overline{3,5,11,25}+\overline{3,5,9,27}+\overline{3,5,7,29}+$ $\overline{9,17,19}+\overline{9,15,21}+\overline{5,19,21}+\overline{9,13,23}+\overline{5,17,23}+\overline{9,11,25}+\overline{5,15,25}+\overline{5,13,27}+$ $\overline{7,9,29}+\overline{5,11,29}+\overline{5,7,33}+\left.\overline{3,9,33}\right|_{45}+\overline{5,9,15,17}+\overline{5,9,13,19}+\overline{5,9,11,21}+$ $\overline{5,7,9,25}+\overline{3,5,9,29}+\left.\overline{5,9,33}\right|_{47}+\overline{3,5,7,9,11,13}+\overline{3,13,15,17}+\overline{7,9,11,21}+$ $\overline{3,5,19,21}+\overline{3,9,13,23}+\overline{3,5,17,23}+\overline{5,7,11,25}+\overline{3,5,15,25}+\overline{23,25}+\overline{21,27}+$ $\overline{3,7,9,29}+\overline{19,29}+\overline{3,5,7,33}+\overline{15,33}+\overline{5,7,9,11,17}+\overline{3,7,9,13,17}+\overline{3,5,11,13,17}+$ $\overline{3,5,9,15,17}+\overline{3,5,9,13,19}+\overline{13,17,19}+\overline{3,5,9,11,21}+\overline{9,19,21}+\overline{3,5,7,9,25}+$ $\overline{9,15,25}+\overline{9,13,27}+\overline{5,17,27}+\overline{9,11,29}+\overline{5,11,33}$

$e_{4}=\left.\overline{3,7,11}\right|_{21}+\overline{5,7,11}+\overline{3,9,11}+\left.\overline{3,7,13}\right|_{23}+\overline{5,9,11}+\overline{5,7,13}+\left.\overline{3,9,13}\right|_{25}+\overline{3,5,7,11}+$ $\overline{7,9,11}+\overline{5,9,13}+\overline{3,11,13}+\left.\overline{3,7,17}\right|_{27}+\overline{3,5,9,11}+\overline{3,5,7,13}+\overline{7,9,13}+\overline{5,11,13}+$ $\overline{5,7,17}+\left.\overline{3,9,17}\right|_{29}+\overline{3,5,9,13}+\left.\overline{5,9,17}\right|_{31}+\overline{5,7,9,11}+\overline{3,5,11,13}+\overline{3,5,7,17}+$ $\overline{9,11,13}+\overline{7,9,17}+\left.\overline{3,13,17}\right|_{33}+\overline{5,7,9,13}+\overline{3,7,11,13}+\overline{3,5,9,17}+\overline{3,5,7,9,11}+$ $\overline{7,11,17}+\overline{5,13,17}+\overline{3,11,21}+\left.\overline{3,7,25}\right|_{35}+\overline{5,7,11,13}+\overline{3,9,11,13}+\overline{3,5,7,9,13}+$ $\overline{9,11,17}+\overline{7,13,17}+\overline{3,15,19}+\overline{5,11,21}+\overline{3,13,21}+\overline{3,11,23}+\overline{5,7,25}+\overline{3,9,25}+$ $\left.\overline{3,7,27}\right|_{37}+\overline{5,7,9,17}+\overline{3,5,13,17}+\overline{5,15,19}+\overline{3,17,19}+\overline{5,13,21}+\overline{3,15,21}+\overline{5,11,23}+$ $\overline{3,13,23}+\overline{5,9,25}+\overline{3,11,25}+\overline{5,7,27}+\overline{3,9,27}+\left.\overline{3,7,29}\right|_{39}+\overline{7,9,11,13}+\overline{5,7,11,17}+$ $\overline{3,7,13,17}+\overline{3,5,11,21}+\overline{3,5,7,25}+\overline{3,5,9,11,13}+\overline{3,5,7,9,17}+\overline{11,13,17}+\overline{5,17,19}+$ $\overline{9,11,21}+\overline{5,15,21}+\overline{5,13,23}+\overline{7,9,25}+\overline{5,11,25}+\overline{5,9,27}+\overline{5,7,29}+\left.\overline{3,9,29}\right|_{41}+$ $\overline{5,9,11,17}+\overline{3,9,13,17}+\overline{3,5,15,19}+\overline{3,5,13,21}+\overline{3,5,11,23}+\overline{3,5,9,25}+\overline{3,5,7,27}+$ $\overline{9,15,19}+\overline{9,13,21}+\overline{3,19,21}+\overline{9,11,23}+\overline{3,17,23}+\overline{3,15,25}+\overline{7,9,27}+\overline{3,13,27}+$ $\overline{5,9,29}+\overline{3,11,29}+\left.\overline{3,7,33}\right|_{43}+\overline{5,9,13,17}+\overline{3,5,17,19}+\overline{3,5,15,21}+\overline{3,5,13,23}+$ $\overline{3,5,11,25}+\overline{3,5,9,27}+\overline{3,5,7,29}+\overline{9,17,19}+\overline{9,15,21}+\overline{5,19,21}+\overline{9,13,23}+$ $\overline{5,17,23}+\overline{9,11,25}+\overline{5,15,25}+\overline{5,13,27}+\overline{7,9,29}+\overline{5,11,29}+\overline{5,7,33}+\left.\overline{3,9,33}\right|_{45}+$ $\overline{7,9,13,17}+\overline{5,11,13,17}+\overline{5,9,11,21}+\overline{5,7,9,25}+\overline{3,5,9,29}+\overline{3,5,9,13,17}+$ $\left.\overline{5,9,33}\right|_{47}+\overline{3,5,7,9,11,13}+\overline{7,11,13,17}+\overline{5,9,15,19}+\overline{5,9,13,21}+\overline{3,11,13,21}+$ $\overline{3,5,19,21}+\overline{5,9,11,23}+\overline{3,5,17,23}+\overline{3,7,13,25}+\overline{3,5,15,25}+\overline{5,7,9,27}+\overline{3,5,13,27}+$ $\overline{3,5,11,29}+\overline{3,5,7,33}+\overline{5,7,9,11,17}+\overline{3,5,9,11,21}+\overline{11,17,21}+\overline{9,19,21}+\overline{9,17,23}+$ $\overline{3,5,7,9,25}+\overline{9,15,25}+\overline{7,17,25}+\overline{9,13,27}+\overline{9,11,29}+\overline{3,17,29}+\overline{7,9,33}+\overline{3,13,33}$ 
$e_{5}=\left.\overline{5,9,13,17}\right|_{45}+\overline{7,9,13,17}+\overline{5,11,13,17}+\overline{5,9,15,17}+\overline{5,9,13,19}+\left.\overline{3,5,9,13,17}\right|_{47}+$ $\overline{7,11,13,17}+\overline{7,9,15,17}+\overline{5,11,15,17}+\overline{7,9,13,19}+\overline{5,11,13,19}+\overline{5,9,15,19}+$ $\overline{5,9,13,21}+\overline{3,7,9,13,17}+\overline{3,5,11,13,17}+\overline{3,5,9,15,17}+\overline{3,5,9,13,19}$

Primitive central idempotents of $\mathbb{F}_{2} S_{n}$ for $n$ even and $n \leq 50$ :

$e_{1}=\left.\overline{1}\right|_{2}+\left.\overline{5}\right|_{6}+\overline{7}+\left.\overline{3,5}\right|_{8}+\left.\overline{9}\right|_{10}+\overline{15}+\overline{7,9}+\overline{5,11}+\left.\overline{3,13}\right|_{16}+\left.\overline{17}\right|_{18}+\left.\overline{5,9,13}\right|_{28}+\overline{7,9,13}+$ $\overline{5,11,13}+\overline{5,9,15}+\left.\overline{3,5,9,13}\right|_{30}+\overline{7,11,13}+\overline{7,9,15}+\overline{5,11,15}+\overline{5,9,17}+\overline{31}+\overline{3,7,9,13}+$ $\overline{3,5,11,13}+\overline{3,5,9,15}+\overline{15,17}+\overline{13,19}+\overline{11,21}+\overline{9,23}+\overline{7,25}+\overline{5,27}+\left.\overline{3,29}\right|_{32}+\overline{9,11,13}+$ $\overline{7,11,15}+\overline{5,13,15}+\overline{7,9,17}+\overline{5,11,17}+\overline{33}+\overline{5,7,9,13}+\overline{3,7,11,13}+\overline{3,7,9,15}+$ $\overline{3,5,11,15}+\left.\overline{3,5,9,17}\right|_{34}+\overline{9,11,15}+\overline{7,13,15}+\overline{7,11,17}+\overline{5,13,17}+\overline{5,7,11,13}+$ $\overline{3,9,11,13}+\overline{5,7,9,15}+\overline{3,5,13,15}+\overline{3,7,9,17}+\left.\overline{3,5,11,17}\right|_{36}+\overline{3,5,7,9,13}+\overline{9,11,17}+$ $\overline{7,13,17}+\overline{5,13,19}+\overline{5,9,23}+\overline{5,7,9,17}+\left.\overline{3,5,13,17}\right|_{38}+\overline{3,5,7,11,13}+\overline{3,5,7,9,15}+$ $\overline{11,13,15}+\overline{7,13,19}+\overline{5,15,19}+\overline{5,13,21}+\overline{7,9,23}+\overline{5,11,23}+\overline{5,9,25}+\overline{7,9,11,13}+$ $\overline{5,9,11,15}+\overline{3,9,13,15}+\overline{3,5,13,19}+\left.\overline{3,5,9,23}\right|_{40}+\overline{3,5,9,11,13}+\overline{3,5,7,9,17}+$ $\overline{11,13,17}+\overline{9,13,19}+\overline{7,15,19}+\overline{5,17,19}+\overline{7,13,21}+\overline{5,15,21}+\overline{7,11,23}+\overline{5,13,23}+$ $\overline{7,9,25}+\overline{5,11,25}+\overline{5,9,11,17}+\overline{3,9,13,17}+\overline{3,7,15,17}+\overline{3,7,13,19}+\overline{3,5,15,19}+$ $\overline{3,7,11,21}+\overline{3,5,13,21}+\overline{3,7,9,23}+\overline{3,5,11,23}+\left.\overline{3,5,9,25}\right|_{42}+\overline{9,15,19}+\overline{7,17,19}+$ $\overline{9,13,21}+\overline{7,15,21}+\overline{9,11,23}+\overline{7,13,23}+\overline{7,11,25}+\overline{5,9,29}+\overline{5,7,15,17}+\overline{3,9,15,17}+$ $\overline{5,7,13,19}+\overline{3,9,13,19}+\overline{3,5,17,19}+\overline{5,7,11,21}+\overline{3,9,11,21}+\overline{3,5,15,21}+\overline{5,7,9,23}+$ $\overline{3,5,13,23}+\overline{3,7,9,25}+\left.\overline{3,5,11,25}\right|_{44}+\overline{9,17,19}+\overline{9,15,21}+\overline{5,19,21}+\overline{9,13,23}+$ $\overline{5,17,23}+\overline{9,11,25}+\overline{5,15,25}+\overline{5,13,27}+\overline{7,9,29}+\overline{5,11,29}+\overline{5,9,31}+\overline{5,9,15,17}+$ $\overline{5,9,13,19}+\overline{5,9,11,21}+\overline{5,7,9,25}+\left.\overline{3,5,9,29}\right|_{46}+\overline{5,7,9,11,15}+\overline{3,7,9,13,15}+$ $\overline{3,5,11,13,15}+\overline{3,5,7,15,17}+\overline{3,5,7,13,19}+\overline{13,15,19}+\overline{3,5,7,11,21}+\overline{7,19,21}+$ $\overline{3,5,7,9,23}+\overline{9,15,23}+\overline{7,17,23}+\overline{7,15,25}+\overline{7,13,27}+\overline{5,15,27}+\overline{7,11,29}+$ $\overline{7,9,31}+\overline{5,11,31}+\overline{5,9,33}+\overline{3,5,7,9,11,13}+\overline{7,9,15,17}+\overline{3,13,15,17}+\overline{5,11,13,19}+$ $\overline{7,9,11,21}+\overline{3,11,13,21}+\overline{3,5,19,21}+\overline{5,9,11,23}+\overline{3,5,17,23}+\overline{3,7,13,25}+$ $\overline{3,5,15,25}+\overline{3,5,13,27}+\overline{3,7,9,29}+\overline{3,5,11,29}+\left.\overline{3,5,9,31}\right|_{48}+\overline{5,7,9,11,17}+$ $3,7,9,13,17+\overline{3,5,11,13,17}+\overline{3,5,9,15,17}+\overline{3,5,9,13,19}+\overline{13,17,19}+\overline{3,5,9,11,21}+$ $\overline{13,15,21}+\overline{9,19,21}+\overline{11,15,23}+\overline{3,5,7,9,25}+\overline{9,13,27}+\overline{7,15,27}+\overline{5,17,27}+\overline{9,11,29}+$ $\overline{5,15,29}+\overline{7,11,31}+\overline{5,13,31}+\overline{7,9,33}+\overline{5,11,33}+\overline{7,11,15,17}+\overline{5,13,15,17}+$ $\overline{7,11,13,19}+\overline{7,9,15,19}+\overline{5,11,15,19}+\overline{7,9,13,21}+\overline{3,11,15,21}+\overline{5,9,13,23}+$ $\overline{3,11,13,23}+3,9,15,23+\overline{5,9,11,25}+\overline{5,7,13,25}+\overline{3,9,13,25}+\overline{3,7,15,25}+$ $\overline{3,7,13,27}+\overline{3,5,15,27}+\overline{5,7,9,29}+\overline{3,7,11,29}+\overline{3,7,9,31}+\overline{3,5,11,31}+\overline{3,5,9,33}$

$e_{2}=\left.\overline{5}\right|_{6}+\overline{7}+\left.\overline{3,5}\right|_{8}+\overline{9}+\left.\overline{3,7}\right|_{10}+\overline{5,7}+\left.\overline{3,9}\right|_{12}+\left.\overline{5,9}\right|_{14}+\overline{3,5,7}+\overline{15}+\left.\overline{5,11}\right|_{16}+\overline{3,5,9}+$ $\overline{17}+\overline{7,11}+\overline{5,13}+\left.\overline{3,15}\right|_{18}+\overline{9,11}+\overline{7,13}+\overline{5,15}+\left.\overline{3,17}\right|_{20}+\overline{5,7,9}+\overline{3,5,13}+\left.\overline{5,17}\right|_{22}+$ $\overline{5,7,11}+\overline{3,7,13}+\overline{3,5,15}+\overline{3,5,7,9}+\overline{11,13}+\left.\overline{9,15}\right|_{24}+\overline{5,9,11}+\overline{3,9,13}+\overline{3,5,17}+$ $\left.\overline{9,17}\right|_{26}+\left.\overline{5,9,13}\right|_{28}+\overline{7,9,13}+\overline{5,11,13}+\overline{5,9,15}+\left.\overline{3,5,9,13}\right|_{30}+\overline{7,11,13}+\overline{3,13,15}+$ $\overline{5,9,17}+\overline{31}+\overline{5,7,9,11}+\overline{3,5,9,15}+\overline{13,19}+\overline{9,23}+\left.\overline{5,27}\right|_{32}+\overline{9,11,13}+\overline{7,11,15}+$ $\overline{5,13,15}+\overline{3,13,17}+\overline{33}+\overline{5,7,9,13}+\overline{3,7,11,13}+\overline{3,7,9,15}+\overline{3,5,11,15}+\overline{3,5,9,17}+$ $\overline{15,19}+\overline{13,21}+\overline{11,23}+\overline{9,25}+\overline{7,27}+\overline{5,29}+\left.\overline{3,31}\right|_{34}+\overline{9,11,15}+\overline{7,13,15}+\overline{7,11,17}+$ $\overline{5,13,17}+\overline{5,7,11,13}+\overline{3,9,11,13}+\overline{5,7,9,15}+\overline{3,5,13,15}+\overline{3,7,9,17}+\overline{3,5,11,17}+$ $\overline{17,19}+\overline{15,21}+\overline{13,23}+\overline{11,25}+\overline{9,27}+\overline{7,29}+\overline{5,31}+\left.\overline{3,33}\right|_{36}+\overline{3,5,7,9,13}+$ $\overline{9,11,17}+\overline{7,13,17}+\overline{5,15,17}+\overline{5,11,21}+\overline{5,7,25}+\overline{3,5,29}+\overline{5,7,9,17}+\overline{3,5,13,17}+$ $\left.\overline{5,33}\right|_{38}+\overline{3,5,7,11,13}+\overline{3,5,7,9,15}+\overline{11,13,15}+\overline{7,15,17}+\overline{5,15,19}+\overline{7,11,21}+$ $\overline{5,13,21}+\overline{5,11,23}+\overline{5,9,25}+\overline{5,7,27}+\overline{3,7,29}+\overline{3,5,31}+\overline{7,9,11,13}+\overline{5,9,11,15}+$ $\overline{3,9,13,15}+\overline{3,5,15,17}+\overline{3,5,11,21}+\overline{19,21}+\overline{17,23}+\overline{3,5,7,25}+\overline{15,25}+\overline{13,27}+$ $\overline{11,29}+\left.\overline{9,31}\right|_{40}+\overline{3,5,9,11,13}+\overline{3,5,7,9,17}+\overline{11,13,17}+\overline{9,15,17}+\overline{7,15,19}+\overline{5,17,19}+$ $\overline{9,11,21}+\overline{7,13,21}+\overline{5,15,21}+\overline{7,11,23}+\overline{5,13,23}+\overline{5,11,25}+\overline{5,9,27}+\overline{3,9,29}+$ $\overline{3,5,33}+\overline{5,9,11,17}+\overline{3,9,13,17}+\overline{3,7,15,17}+\overline{3,7,13,19}+\overline{3,5,15,19}+\overline{3,7,11,21}+$ $\overline{3,5,13,21}+\overline{3,7,9,23}+\overline{3,5,11,23}+\overline{3,5,9,25}+\left.\overline{9,33}\right|_{42}+\overline{9,15,19}+\overline{7,17,19}+\overline{9,13,21}+$ $\overline{7,15,21}+\overline{9,11,23}+\overline{7,13,23}+\overline{7,11,25}+\overline{5,9,29}+\overline{5,7,15,17}+\overline{3,9,15,17}+\overline{5,7,13,19}+$ $\overline{3,9,13,19}+\overline{3,5,17,19}+\overline{5,7,11,21}+\overline{3,9,11,21}+\overline{3,5,15,21}+\overline{5,7,9,23}+\overline{3,5,13,23}+$ $\overline{3,7,9,25}+\left.\overline{3,5,11,25}\right|_{44}+\overline{9,17,19}+\overline{9,15,21}+\overline{5,19,21}+\overline{9,13,23}+\overline{5,17,23}+\overline{9,11,25}+$ $\overline{5,15,25}+\overline{5,13,27}+\overline{7,9,29}+\overline{5,11,29}+\overline{5,9,31}+5,9,15,17+\overline{5,9,13,19}+\overline{5,9,11,21}+$ $\overline{5,7,9,25}+\left.\overline{3,5,9,29}\right|_{46}+\overline{5,7,9,11,15}+\overline{3,7,9,13,15}+\overline{3,5,11,13,15}+\overline{3,5,7,15,17}+$ $\overline{3,5,7,13,19}+\overline{3,5,7,11,21}+\overline{11,15,21}+\overline{7,19,21}+\overline{3,5,7,9,23}+\overline{7,17,23}+\overline{7,13,27}+$ $\overline{7,11,29}+\overline{3,15,29}+\overline{3,13,31}+\overline{5,9,33}+\overline{3,5,7,9,11,13}+\overline{5,11,15,17}+\overline{7,9,13,19}+$ $\overline{3,5,19,21}+\overline{3,9,13,23}+\overline{3,5,17,23}+\overline{5,7,11,25}+\overline{3,5,15,25}+\overline{23,25}+\overline{5,7,9,27}+$ $\overline{21,27}+\overline{19,29}+\overline{3,5,9,31}+\left.\overline{17,31}\right|_{48}+\overline{5,7,9,11,17}+\overline{3,7,9,13,17}+\overline{3,5,11,13,17}+$ $\overline{3,5,9,15,17}+\overline{3,5,9,13,19}+\overline{3,5,9,11,21}+\overline{13,15,21}+\overline{11,17,21}+\overline{9,19,21}+$ $\overline{11,15,23}+\overline{9,17,23}+\overline{3,5,7,9,25}+\overline{7,17,25}+\overline{9,13,27}+\overline{7,15,27}+\overline{9,11,29}+\overline{5,15,29}+$ $\overline{3,17,29}+\overline{7,11,31}+\overline{5,13,31}+\overline{3,13,33}+\overline{7,11,15,17}+\overline{5,13,15,17}+\overline{7,11,13,19}+$ $\overline{7,9,15,19}+\overline{5,11,15,19}+\overline{7,9,13,21}+\overline{3,11,15,21}+\overline{5,9,13,23}+\overline{3,11,13,23}+$ $\overline{3,9,15,23}+\overline{5,9,11,25}+\overline{5,7,13,25}+\overline{3,9,13,25}+\overline{3,7,15,25}+\overline{3,7,13,27}+\overline{3,5,15,27}+$ $\overline{5,7,9,29}+\overline{3,7,11,29}+\overline{3,7,9,31}+\overline{3,5,11,31}+\overline{3,5,9,33}+\overline{17,33}$ 
$=\left.\overline{3,7}\right|_{10}+\overline{5,7}+\left.\overline{3,9}\right|_{12}+\left.\overline{5,9}\right|_{14}+\overline{3,5,7}+\overline{7,9}+\left.\overline{3,13}\right|_{16}+\overline{3,5,9}+\overline{7,11}+\overline{5,13}+$ $\left.\overline{3,15}\right|_{18}+\overline{9,11}+\overline{7,13}+\overline{5,15}+\left.\overline{3,17}\right|_{20}+\overline{5,7,9}+\overline{3,5,13}+\left.\overline{5,17}\right|_{22}+\overline{5,7,11}+$ $\overline{3,7,13}+\overline{3,5,15}+\overline{3,5,7,9}+\overline{11,13}+\left.\overline{9,15}\right|_{24}+\overline{5,9,11}+\overline{3,9,13}+\overline{3,5,17}+\left.\overline{9,17}\right|_{26}+$ $\left.\overline{5,9,13}\right|_{28}+\overline{7,9,13}+\overline{5,11,13}+\overline{5,9,15}+\left.\overline{3,5,9,13}\right|_{30}+\overline{7,11,13}+\overline{3,13,15}+\overline{5,9,17}+$ $\overline{5,7,9,11}+\overline{3,5,9,15}+\overline{15,17}+\overline{11,21}+\overline{7,25}+\left.\overline{3,29}\right|_{32}+\overline{9,11,13}+\overline{7,11,15}+\overline{5,13,15}+$ $\overline{3,13,17}+\overline{5,7,9,13}+\overline{3,7,11,13}+\overline{3,7,9,15}+\overline{3,5,11,15}+\overline{3,5,9,17}+\overline{15,19}+\overline{13,21}+$ $\overline{11,23}+\overline{9,25}+\overline{7,27}+\overline{5,29}+\left.\overline{3,31}\right|_{34}+\overline{9,11,15}+\overline{7,13,15}+\overline{7,11,17}+\overline{5,13,17}+$ $\overline{5,7,11,13}+\overline{3,9,11,13}+\overline{5,7,9,15}+\overline{3,5,13,15}+\overline{3,7,9,17}+\overline{3,5,11,17}+\overline{17,19}+$ $\overline{15,21}+\overline{13,23}+\overline{11,25}+\overline{9,27}+\overline{7,29}+\overline{5,31}+\left.\overline{3,33}\right|_{36}+\overline{3,5,7,9,13}+\overline{9,11,17}+$ $\overline{7,13,17}+\overline{5,15,17}+\overline{5,11,21}+\overline{5,7,25}+\overline{3,5,29}+\overline{5,7,9,17}+\overline{3,5,13,17}+\left.\overline{5,33}\right|_{38}+$ $\overline{3,5,7,11,13}+\overline{3,5,7,9,15}+\overline{11,13,15}+\overline{7,15,17}+\overline{5,15,19}+\overline{7,11,21}+\overline{5,13,21}+$ $\overline{5,11,23}+\overline{5,9,25}+\overline{5,7,27}+\overline{3,7,29}+\overline{3,5,31}+\overline{7,9,11,13}+\overline{5,9,11,15}+\overline{3,9,13,15}+$ $\overline{3,5,15,17}+\overline{3,5,11,21}+\overline{19,21}+\overline{17,23}+\overline{3,5,7,25}+\overline{15,25}+\overline{13,27}+\overline{11,29}+\left.\overline{9,31}\right|_{40}+$ $\overline{3,5,9,11,13}+\overline{3,5,7,9,17}+\overline{11,13,17}+\overline{9,15,17}+\overline{7,15,19}+\overline{5,17,19}+\overline{9,11,21}+$ $\overline{7,13,21}+\overline{5,15,21}+\overline{7,11,23}+\overline{5,13,23}+\overline{5,11,25}+\overline{5,9,27}+\overline{3,9,29}+\overline{3,5,33}+$ $\overline{5,9,11,17}+\overline{3,9,13,17}+\overline{3,7,15,17}+\overline{3,7,13,19}+\overline{3,5,15,19}+\overline{3,7,11,21}+\overline{3,5,13,21}+$ $\overline{3,7,9,23}+\overline{3,5,11,23}+\overline{3,5,9,25}+\left.\overline{9,33}\right|_{42}+\overline{9,15,19}+\overline{7,17,19}+\overline{9,13,21}+\overline{7,15,21}+$ $\overline{9,11,23}+\overline{7,13,23}+\overline{7,11,25}+\overline{5,9,29}+\overline{5,7,15,17}+\overline{3,9,15,17}+\overline{5,7,13,19}+$ $\overline{3,9,13,19}+\overline{3,5,17,19}+\overline{5,7,11,21}+\overline{3,9,11,21}+\overline{3,5,15,21}+\overline{5,7,9,23}+\overline{3,5,13,23}+$ $\overline{3,7,9,25}+\left.\overline{3,5,11,25}\right|_{44}+\overline{9,17,19}+\overline{9,15,21}+\overline{5,19,21}+\overline{9,13,23}+\overline{5,17,23}+\overline{9,11,25}+$ $5,15,25+\overline{5,13,27}+\overline{7,9,29}+\overline{5,11,29}+\overline{5,9,31}+\overline{5,9,15,17}+\overline{5,9,13,19}+\overline{5,9,11,21}+$ $\overline{5,7,9,25}+\left.\overline{3,5,9,29}\right|_{46}+\overline{5,7,9,11,15}+\overline{3,7,9,13,15}+\overline{3,5,11,13,15}+\overline{3,5,7,15,17}+$ $\overline{3,5,7,13,19}+\overline{3,5,7,11,21}+\overline{11,15,21}+\overline{7,19,21}+\overline{3,5,7,9,23}+\overline{7,17,23}+\overline{7,13,27}+$ $\overline{7,11,29}+\overline{3,15,29}+\overline{3,13,31}+\overline{5,9,33}+\overline{3,5,7,9,11,13}+\overline{5,11,15,17}+\overline{7,9,13,19}+$ $\overline{3,5,19,21}+\overline{3,9,13,23}+\overline{3,5,17,23}+\overline{5,7,11,25}+\overline{3,5,15,25}+\overline{23,25}+\overline{5,7,9,27}+$ $\overline{21,27}+\overline{19,29}+\overline{3,5,9,31}+\left.\overline{17,31}\right|_{48}+\overline{5,7,9,11,17}+\overline{3,7,9,13,17}+\overline{3,5,11,13,17}+$ $\overline{3,5,9,15,17}+\overline{3,5,9,13,19}+\overline{3,5,9,11,21}+\overline{13,15,21}+\overline{11,17,21}+\overline{9,19,21}+$ $\overline{11,15,23}+\overline{9,17,23}+\overline{3,5,7,9,25}+\overline{7,17,25}+\overline{9,13,27}+\overline{7,15,27}+\overline{9,11,29}+\overline{5,15,29}+$ $\overline{3,17,29}+\overline{7,11,31}+\overline{5,13,31}+\overline{3,13,33}+\overline{7,11,15,17}+\overline{5,13,15,17}+\overline{7,11,13,19}+$ $\overline{7,9,15,19}+\overline{5,11,15,19}+\overline{7,9,13,21}+\overline{3,11,15,21}+\overline{5,9,13,23}+\overline{3,11,13,23}+$ $\overline{3,9,15,23}+\overline{5,9,11,25}+\overline{5,7,13,25}+\overline{3,9,13,25}+\overline{3,7,15,25}+\overline{3,7,13,27}+\overline{3,5,15,27}+$ $\overline{5,7,9,29}+\overline{3,7,11,29}+\overline{3,7,9,31}+\overline{3,5,11,31}+\overline{3,5,9,33}+\overline{17,33}$

$e_{4}=\left.\overline{5,9,13}\right|_{28}+\overline{7,9,13}+\overline{5,11,13}+\overline{5,9,15}+\left.\overline{3,5,9,13}\right|_{30}+\overline{7,11,13}+\overline{7,9,15}+\overline{5,11,15}+$ $\overline{5,9,17}+\overline{3,7,9,13}+\overline{3,5,11,13}+\left.\overline{3,5,9,15}\right|_{32}+\overline{9,11,13}+\overline{7,11,15}+\overline{5,13,15}+\overline{7,9,17}+$ $\overline{5,11,17}+\overline{5,7,9,13}+\overline{3,7,11,13}+\overline{3,7,9,15}+\overline{3,5,11,15}+\left.\overline{3,5,9,17}\right|_{34}+\overline{9,11,15}+$ $\overline{7,13,15}+\overline{7,11,17}+\overline{5,13,17}+\overline{5,7,11,13}+\overline{3,9,11,13}+\overline{5,7,9,15}+\overline{3,7,11,15}+$ $\overline{3,5,13,15}+\overline{3,7,9,17}+\left.\overline{3,5,11,17}\right|_{36}+\overline{3,5,7,9,13}+\overline{9,11,17}+\overline{7,13,17}+\overline{5,13,19}+$ $5,9,23+\overline{5,7,11,15}+\overline{3,9,11,15}+\overline{3,7,13,15}+\overline{5,7,9,17}+\overline{3,7,11,17}+\left.\overline{3,5,13,17}\right|_{38}+$ $\overline{3,5,7,11,13}+\overline{3,5,7,9,15}+\overline{11,13,15}+\overline{7,13,19}+\overline{5,15,19}+\overline{5,13,21}+\overline{7,9,23}+$ $\overline{5,11,23}+\overline{5,9,25}+\overline{7,9,11,13}+\overline{5,7,13,15}+\overline{5,7,11,17}+\overline{3,9,11,17}+\overline{3,7,13,17}+$ $3,5,13,19+\left.\overline{3,5,9,23}\right|_{40}+\overline{3,5,9,11,13}+\overline{3,5,7,11,15}+\overline{3,5,7,9,17}+\overline{11,13,17}+$ $\overline{9,13,19}+\overline{7,15,19}+\overline{5,17,19}+\overline{7,13,21}+\overline{5,15,21}+\overline{7,11,23}+\overline{5,13,23}+\overline{7,9,25}+$ $\overline{5,11,25}+\overline{7,9,11,15}+\overline{5,9,13,15}+\overline{3,11,13,15}+\overline{5,7,13,17}+\overline{3,7,13,19}+\overline{3,5,15,19}+$ $\overline{3,5,13,21}+\overline{3,7,9,23}+\overline{3,5,11,23}+\left.\overline{3,5,9,25}\right|_{42}+\overline{3,5,9,11,15}+\overline{3,5,7,13,15}+$ $\overline{3,5,7,11,17}+\overline{9,15,19}+\overline{7,17,19}+\overline{9,13,21}+\overline{7,15,21}+\overline{9,11,23}+\overline{7,13,23}+\overline{7,11,25}+$ $5,9,29+\overline{7,9,13,15}+\overline{5,11,13,15}+\overline{7,9,11,17}+\overline{5,9,13,17}+\overline{3,11,13,17}+\overline{5,7,13,19}+$ $\overline{3,9,13,19}+\overline{3,7,15,19}+\overline{3,5,17,19}+\overline{3,7,13,21}+\overline{3,5,15,21}+\overline{5,7,9,23}+\overline{3,7,11,23}+$ $\overline{3,5,13,23}+\overline{3,7,9,25}+\left.\overline{3,5,11,25}\right|_{44}+\overline{3,5,9,13,15}+\overline{3,5,9,11,17}+\overline{3,5,7,13,17}+$ $\overline{9,17,19}+\overline{9,15,21}+\overline{5,19,21}+\overline{9,13,23}+\overline{5,17,23}+\overline{9,11,25}+\overline{5,15,25}+\overline{5,13,27}+$ $\overline{7,9,29}+\overline{5,11,29}+\overline{5,9,31}+\overline{7,9,13,17}+\overline{5,11,13,17}+\overline{5,9,13,19}+\overline{5,7,15,19}+$ $\overline{3,9,15,19}+\overline{3,7,17,19}+\overline{5,7,13,21}+\overline{3,9,13,21}+\overline{3,7,15,21}+\overline{5,7,11,23}+\overline{3,9,11,23}+$ $\overline{3,7,13,23}+\overline{5,7,9,25}+\overline{3,7,11,25}+\left.\overline{3,5,9,29}\right|_{46}+\overline{3,7,9,13,15}+\overline{3,5,9,13,17}+$ $\overline{3,5,7,13,19}+\overline{13,15,19}+\overline{7,19,21}+\overline{3,5,7,9,23}+\overline{9,15,23}+\overline{7,17,23}+\overline{7,15,25}+$ $\overline{7,13,27}+\overline{5,15,27}+\overline{7,11,29}+\overline{7,9,31}+\overline{5,11,31}+\overline{5,9,33}+\overline{3,5,7,9,11,13}+$ $9,11,13,15+5,11,13,19+5,9,15,19+5,7,17,19+\overline{3,9,17,19}+\overline{5,9,13,21}+$ $\overline{5,7,15,21}+\overline{3,9,15,21}+\overline{3,5,19,21}+\overline{5,7,13,23}+\overline{3,9,13,23}+\overline{3,5,17,23}+\overline{5,7,11,25}+$ $\overline{3,9,11,25}+\overline{3,5,15,25}+\overline{3,5,13,27}+\overline{3,7,9,29}+\overline{3,5,11,29}+\overline{3,5,9,31}_{48}+$ $\overline{5,7,9,13,15}+\overline{3,7,11,13,15}+\overline{3,7,9,13,17}+\overline{3,5,9,13,19}+\overline{3,5,7,15,19}+\overline{13,17,19}+$ $\overline{3,5,7,13,21}+\overline{13,15,21}+\overline{9,19,21}+\overline{3,5,7,11,23}+\overline{11,15,23}+\overline{3,5,7,9,25}+\overline{9,13,27}+$ $\overline{7,15,27}+\overline{5,17,27}+\overline{9,11,29}+\overline{5,15,29}+\overline{7,11,31}+\overline{5,13,31}+\overline{7,9,33}+\overline{5,11,33}+$ $\overline{3,5,7,9,11,15}+\overline{9,11,13,17}+\overline{7,11,13,19}+\overline{5,11,15,19}+\overline{3,13,15,19}+\overline{5,9,17,19}+$ $\overline{5,11,13,21}+\overline{5,9,15,21}+\overline{3,7,19,21}+\overline{7,9,11,23}+\overline{3,9,15,23}+\overline{3,7,17,23}+$ $\overline{3,7,15,25}+\overline{3,7,13,27}+\overline{3,5,15,27}+\overline{5,7,9,29}+\overline{3,7,11,29}+\overline{3,7,9,31}+\overline{3,5,11,31}+$ $\overline{3,5,9,33}$ 
For alternating groups $\mathbb{F}_{4}$ is always a splitting field. The primitive central idempotents of $\mathbb{F}_{4} A_{n}$ are the primitive central idempotents of $\mathbb{F}_{2} S_{n}$ except for one case: If $n=\frac{m(m+1)}{2}$, then there is an idempotent $e=C^{+}$of $\mathbb{F}_{2} S_{n}$, where $C$ is the conjugacy class corresponding to the partition $(2 m-1,2 m-5,2 m-9, \ldots)$ of $n$. This idempotent splits in two primitive central idempotents $f_{1}$ and $f_{2}$ of $\mathbb{F}_{4} A_{n}$. We computed these two idempotents. If a class $C$ of $S_{n}$ splits in two conjugacy classes of $A_{n}$, then we write $C_{-}$and $C_{+}$for the $A_{n}$-classes. $\zeta$ denotes a generator of $\mathbb{F}_{4}$ over $\mathbb{F}_{2}$. To save space we only write $f_{1}$, the second idempotent $f_{2}$ can easily be computed via $f_{2}=f_{1}+\overline{2 m-1,2 m-5,2 m-9, \ldots+}+\overline{2 m-1,2 m-5,2 m-9, \ldots-}$.

\begin{tabular}{|c|c|}
\hline & \\
\hline 3 & $\overline{1}+\zeta^{2} \cdot \overline{3_{+}}+\zeta \cdot \overline{3_{-}}$ \\
\hline 6 & $\overline{3}+\zeta^{2} \cdot \overline{5_{+}}+\zeta \cdot \overline{5_{-}}+\overline{3^{2}}$ \\
\hline 10 & $+\overline{3,5}+\overline{3^{3}}+\overline{5^{2}}+\zeta^{2} \cdot \overline{3,7_{+}}+\zeta \cdot \overline{3,7_{-}}$ \\
\hline 15 & $\overline{5,7}+\overline{3,9}+\overline{3^{3}, 5}+\overline{7^{2}}+\zeta \cdot \overline{5,9_{+}}+\zeta^{2} \cdot \overline{5,9_{-}}+\overline{5^{3}}+\overline{3,5,7_{+}}+\overline{3,5,7_{-}}+\overline{3^{2}, 9}$ \\
\hline 21 & 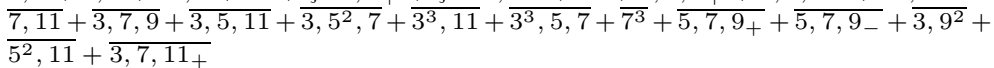 \\
\hline 28 & 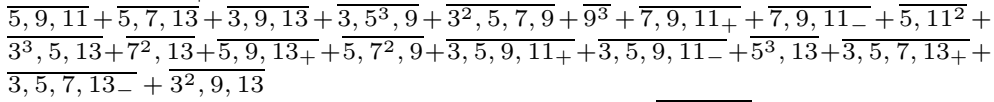 \\
\hline 36 & 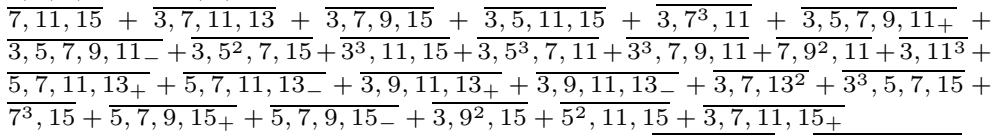 \\
\hline 45 & 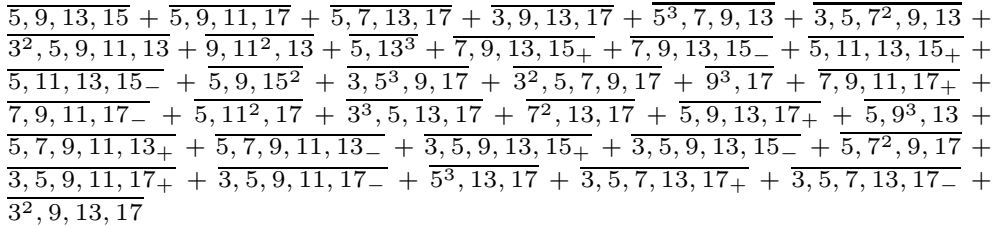 \\
\hline 55 & 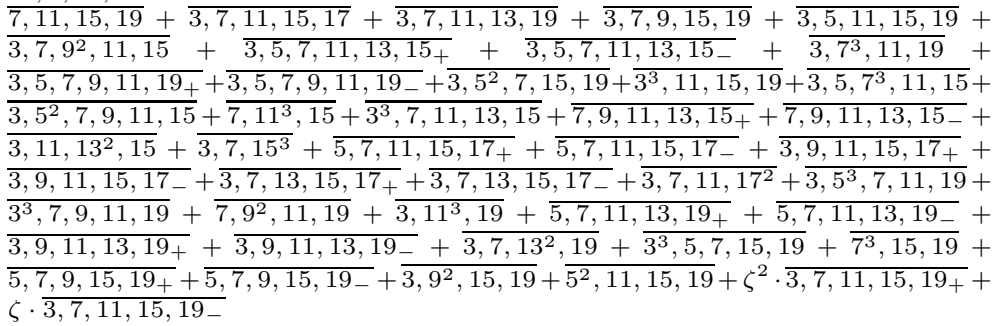 \\
\hline
\end{tabular}




\section{REFERENCES}

[1] C. Curtis and I. Reiner, 'Representation Theory of Finite Groups and Associative Algebras', Interscience Publishers, New York, London, Sydney, 1962. MR0144979 (26:2519)

[2] The GAP Group, GAP — Groups, Algorithms, and Programming, Version 4.4; 2005. (http://www.gap-system.org)

[3] R. Gow, 'Real 2-Blocks of $S_{n}, A_{n}$ and Their Double Covers', The Arcata Conference on Representations of Finite Groups, Proc. Symposia Pure Math. 47 (1987), 421-429. MR 933379 (89g:20013)

[4] B. Huppert, 'Character Theory of Finite Groups', Walter de Gruyter, Berlin, New York, 1998. MR1645304 (99j:20011)

[5] B. Huppert, 'Endliche Gruppen I', Springer, Berlin, Heidelberg, New York, 1967. MR0224703 $(37: 302)$

[6] B. Huppert and N. Blackburn, 'Finite Groups II', Springer, Berlin, Heidelberg, New York, 1982. MR650245 (84i:20001a)

[7] G. James and A. Kerber, 'The Representation Theory of the Symmetric Group', Encyclopedia of Mathematics and its Applications, Addison-Wesley, London, 1981. MR644144 (83k:20003)

[8] G. Karpilovsky, 'Structure of Blocks of Group Algebras', Longman Scientific \& Technical, Harlow, 1987. MR.914150 (88k:20001)

[9] A. Kerber, 'Representations of Permutation Groups I', Lecture Notes in Mathematics 240, Springer-Verlag, Berlin, Heidelberg, New York, 1971. MR0325752 (48:4098)

[10] A. Kerber, A. Kohnert and A. Lascoux, 'SYMMETRICA, an object oriented computeralgebra system for the symmetric group', J. Symbolic Comput. 14 (1992), 195-203. MR:1187231 (93g:68062)

[11] B. Külshammer, 'Group-theoretical descriptions of ring-theoretical invariants of group algebras', Progr. Math. 95 (1991), 425-442. MR1112173 (92d:16037)

[12] H. Meyer, 'Konjugationsklassensummen in endlichen Gruppenringen', thesis, Bayreuth. Math. Schr. 66, Bayreuth, 2002. MR1953638 (2003k:20008)

[13] H. Meyer, 'Finite splitting fields of normal subgroups', Arch. Math. 83 (2004) 97-101. MR2104936 (2005h:20016)

[14] J. Murray, 'Squares in the Centre of the Group Algebra of a Symmetric Group', Bull. London Math. Soc. 34 (2002) 155-164. MR.1874081(2002i:20017)

[15] G. Navarro, Characters and Blocks of Finite Groups, Cambridge University Press, Cambridge, 1998. MR.1632299 (2000a:20018)

[16] J. Olsson, 'Lower Defect Groups in Symmetric Groups', J. Alg. 104 (1986) 37-56. MR865885 (87m:20039)

[17] M. Suzuki, 'Group Theory I', Springer-Verlag, New York, Berlin, Heidelberg, Tokyo, 1982. MR648772 (82k:20001c)

Mathematisches Institut, Universität Bayreuth, 95440 Bayreuth, Germany

E-mail address: harald.meyer@uni-bayreuth.de 